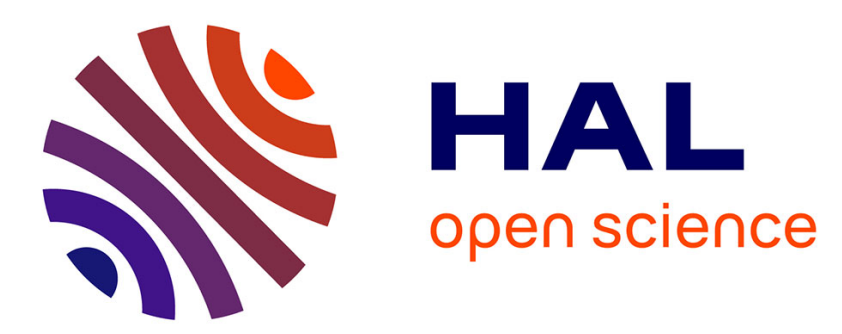

\title{
Particle transport due to energetic-particle-driven geodesic acoustic modes
}

\author{
David Zarzoso, D Del-Castillo-Negrete, D F Escande, Y. Sarazin, X. Garbet, \\ V. Grandgirard, C. Passeron, G. Latu, S. Benkadda
}

\section{- To cite this version:}

David Zarzoso, D Del-Castillo-Negrete, D F Escande, Y. Sarazin, X. Garbet, et al.. Particle transport due to energetic-particle-driven geodesic acoustic modes. Nuclear Fusion, 2018, 58 (10), 10.1088/17414326/aad785 . hal-01899139

\section{HAL Id: hal-01899139 \\ https://hal.science/hal-01899139}

Submitted on 19 Oct 2018

HAL is a multi-disciplinary open access archive for the deposit and dissemination of scientific research documents, whether they are published or not. The documents may come from teaching and research institutions in France or abroad, or from public or private research centers.
L'archive ouverte pluridisciplinaire HAL, est destinée au dépôt et à la diffusion de documents scientifiques de niveau recherche, publiés ou non, émanant des établissements d'enseignement et de recherche français ou étrangers, des laboratoires publics ou privés. 


\title{
Particle transport due to energetic-particle-driven geodesic acoustic modes
}

\author{
D. Zarzoso ${ }^{1}$, D. Del-Castillo-Negrete ${ }^{2}$, D. F. Escande ${ }^{1}$, Y.

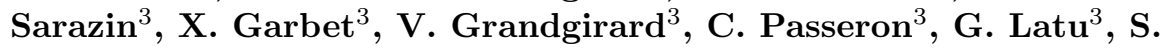 \\ Benkadda $^{1}$ \\ 1 Aix-Marseille Université, CNRS, PIIM, UMR 7345 Marseille, France. \\ 2 Oak Ridge National Laboratory, Oak Ridge, Tennessee 37831-8071, USA \\ ${ }^{3}$ CEA, IRFM, 13108 Saint-Paul-lez-Durance, France.
}

\begin{abstract}
The transport of particles in the presence of energetic geodesic acoustic modes (EGAMs) is analysed by means of full- $f$ global gyro-kinetic simulations using the multispecies version of the GYSELA code and a test-particle tracking post-treatment that solves the equations of motion of passive gyro-centres embedded in the self-consistent EGAM potential obtained from GySELA simulations. It is found that EGAMs induce transport of particles that eventually results in counter-passing particle losses modulated at the EGAM frequency. A detailed analysis of the trajectories of test gyro-centres is performed and evidences of the interaction between the EGAM island and the region of magnetically trapped particles (trapping cone) is observed. In particular, we report for the first time on the complex interaction between the stochastic separatrix of the EGAM island and the $\mathrm{X}$-point of the trapping cone, creating a channel for the transport of particles from $v_{\|}<0$ to $v_{\|}>0$ regions. Therefore, for the cases analysed in this work, co-injection of energetic particles might lead to a significant reduction of EGAM-induced losses. This result opens up new perspectives for further studies of the selection of energetic particle injection in order to minimize the losses due to EGAMs.
\end{abstract}




\section{Introduction}

Energetic particles (EP) are ubiquitous in both laboratory and astrophysical plasmas. By definition, they exhibit velocities much larger than the thermal velocity of the bulk plasma, which is characterized by a Maxwellian distribution function. The presence of a substantial population of particles at high energies leads to gradients in phase space, which may result in instabilities called energetic particle modes (EPM). In this paper, we explore a class of EPM called energetic geodesic acoustic modes (EGAMs), characteristic of toroidal devices, such as tokamaks, but the present work can be extended to other complex systems. As reported in [1,2], EGAMs are born from either the standard geodesic acoustic modes (GAMs) [3] or from an initially Landau damped mode that becomes unstable in the presence of a population of energetic particles [4]. As the standard GAMs, EGAMs have the particularity to be axi-symmetric modes, meaning that there is no dependence on the toroidal angle, and they correspond to the oscillating component of the zonal flows. Because of the beneficial impact that zonal flows can have on turbulence, GAMs have received much attention in the last years, and EGAMs have been seen as a possible way to control turbulence by injection of energetic particles. EGAMs have been observed in many experimental devices [5-8], modelled in numerical simulations [1,9-14] and explained in analytic theory [1, 2, 4, 9, 15-22]. Since they are axisymmetric modes, the toroidal canonical momentum, $P_{\varphi}$, is conserved. For low toroidal velocities the toroidal canonical momentum can be approximated by the poloidal flux, which is directly related to the radial position. Therefore, under this assumption, axisymmetric modes (and in particular EGAMs) are not expected to induce a significant radial transport of particles. However, for energetic particles, the contribution of the toroidal velocity can no longer be neglected in the toroidal canonical momentum. In this case, any modification of the toroidal velocity will result in a substantial variation of the radial position, in virtue of the conservation of $P_{\varphi}$. We should note that thermal particles can also be transported by this mechanism, since the effect comes from the modification of the toroidal velocity. Nevertheless, energetic particles that are thermalized will explore a region in phase space that is wider than that explored by thermal particles. The losses of particles due to EGAMs were first observed in DIII-D discharges [7], where $10 \%-15 \%$ drops in the neutron emission occurred, indicative of strong beam ion redistribution and even losses. It was speculated that EGAMs might modify the pitch angle of counter-passing particles, resulting in unconfined trapped particles. This was later corroborated in [8] by comparison between a DIII$\mathrm{D}$ discharge, where fast-ion loss detector (FILD) [23] data were available, and numerical results from full orbit simulation code SPIRAL [24]. Also, a recent work provides analytical calculation of the effect of the EGAM on well passing particles that are trapped in the EGAM island and subsequent impact on the neutron emission [25]. However, further systematic analysis of particle transport in phase space for all classes of particles in the presence of EGAMs has so far not been conducted, nor the fundamental physics for the trapping of particles has been analysed in detail. In this paper we report for the first time on evidences of particle transport using data from global gyro-kinetic full-f Gysela code [26]. In particular, we show that an interaction between the magnetically trapped particles region and the EGAM island is possible, creating a chaotic region in phase space, allowing the losses of particles by interaction with the $\mathrm{X}$-point of the trapping cone. For this purpose a testparticle tracking post-treatment has been developed, which solves numerically the equations of motion of gyro-centres in a tokamak, as done in GYSELA code, with the only assumption that all fluctuations are axisymmetric. The remainder of this paper is structured as follows. In section 2 we give a brief description of the GYSELA code and of the EP simulations used to observe and analyse the linear excitation and nonlinear saturation of EGAMs. Section 3 is devoted to the description of the newly developed diagnostic used to integrate the particle trajectories. In section 4 we analyse in detail the transport of particles in the presence of EGAMs, their losses as well as the interaction between the EGAM island and the trapping cone. Conclusions are given in section 5 . 


\section{Brief description of GYSELA code and excitation and nonlinear saturation of EGAMs with two kinetic ion species}

Regarding Vlasov equation, Gysela [26] is a full- $f$ global flux-driven gyro-kinetic code that was initially developed for the analysis of electrostatic turbulence in tokamaks. The code solves in a self-consistent way the Vlasov gyro-kinetic equation coupled to the quasi-neutrality condition. Later, the capabilities to add energetic particles using one single kinetic ion species were reported within the framework of the linear excitation of EGAMs and their nonlinear saturation [9]. Moreover, the implementation of a simplified energetic particle source allowed the study of the nonlinear interaction between EGAMs and iontemperature gradient turbulence $[10,14,27]$. Recently, GYSELA was upgraded to have the possibility to introduce two kinetic ion species. Exploiting this new feature, EGAMs have been excited in GySELA using one kinetic species for thermal particles and one kinetic species for energetic particles. For this purpose, the thermal population is modelled by a centred Maxwellian

$F_{\mathrm{th}}=\frac{n_{\mathrm{th}}}{\left(2 \pi T_{\mathrm{th}} / m_{\mathrm{th}}\right)^{3 / 2}} \mathrm{e}^{-\frac{m_{\mathrm{th}} v_{\|}^{2}+2 \mu_{\mathrm{th}} B}{2 T_{\mathrm{th}}}}$

whereas the energetic particle population is modelled by a double shifted Maxwellian in parallel velocity

$$
\begin{aligned}
F_{\mathrm{EP}}= & \frac{1}{2} \frac{n_{\mathrm{EP}}}{\left(2 \pi T_{\mathrm{EP}} / m_{\mathrm{EP}}\right)^{3 / 2}}\left(\mathrm{e}^{-\frac{m_{\mathrm{EP}}\left(v_{\|}-v_{\mathrm{EP}}\right)^{2}+2 \mu_{\mathrm{EP}} B}{2 T_{\mathrm{EP}}}}\right. \\
& \left.+\mathrm{e}^{-\frac{m_{\mathrm{EP}}\left(v_{\|}+v_{\mathrm{EP}}\right)^{2}+2 \mu_{\mathrm{EP}} B}{2 T_{\mathrm{EP}}}}\right)
\end{aligned}
$$

In the previous expressions, $n_{s}, T_{s}, m_{s}$ and $\mu_{s}$ are, for each species $s$, the density, temperature, mass and magnetic moment, respectively. Subscripts th and EP stand for thermal and energetic particles, respectively. The thermal and energetic particle densities satisfy quasi-neutrality condition $Z_{\mathrm{th}} n_{\mathrm{th}}+Z_{\mathrm{EP}} n_{\mathrm{EP}}=n_{e}$, where $Z_{s}$ is the charge number of species $s$ and $n_{e}$ the total plasma density. Please, note that the choice of energetic particle distribution function is based on verification purposes against analytic theory. Nevertheless, as shown in [9], the basic features of the EGAM are not significantly modified when using a more experiment-relevant distribution function and therefore the results reported in the following can be generalized. We have performed a scan on the fraction, mass and charge of energetic particles using deuterium as thermal population. The linear excitation of EGAM has been verified against theoretical predictions [2]. This is shown in figure 1 , where lines represent theoretical predictions from [2] and symbols represent values measured from GyselA simulations. Both the
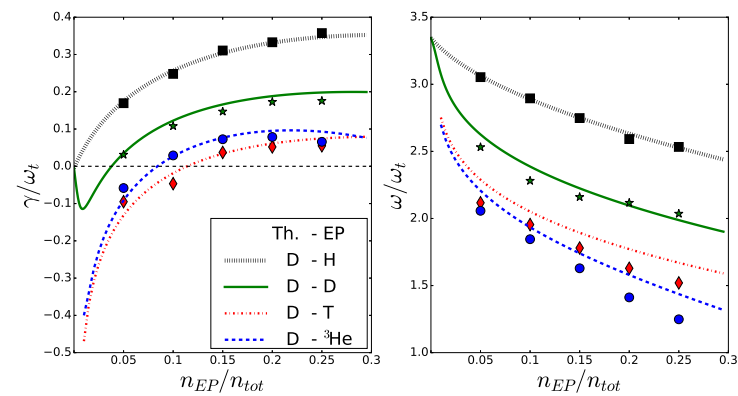

Figure 1: Frequency (left) and growth rate (right) of EGAM as predicted in [2] (lines) and as measured from GysELA simulations (symbols). The scan is performed using the same species for the thermal population and modifying the mass and the charge for the energetic particle population, as done in [2].

growth rate and the real frequency are normalized to the transit frequency of passing particles as defined in [2], $\omega_{t}=\left(q R_{0}\right)^{-1} \sqrt{2 T_{\mathrm{th}} / m_{t h}}$. For the simulations reported in that figure, all the radial profiles are flat, the safety factor is set to $q=1.8$, the ion Larmor radius normalized to the minor radius is $\rho_{\star}=1 / 128$, the temperatures are chosen so that $T_{\mathrm{th}}=T_{\mathrm{EP}}=$ $T_{e}$ and the energetic particle mean velocity is set to $v_{\mathrm{EP}}=4 v_{\mathrm{th}, \mathrm{EP}}$, where $v_{\mathrm{th}, \mathrm{EP}}$ is the thermal velocity of energetic particles defined as $v_{\mathrm{th}, \mathrm{EP}}=\sqrt{T_{\mathrm{EP}} / m_{\mathrm{EP}}}$.

The nonlinear saturation of EGAMs was analysed in [9], where the flattening of the equilibrium distribution function was evidenced by conveniently averaging the full distribution function over flux surfaces. However, in that work thermal and energetic particle distribution functions were indistinguishable. Also, using different distribution functions for thermal and energetic particles allows one to determine to what extent the excitation of EGAMs by energetic particles affects the thermal population. In particular, the existence of higher order resonances can lead to a significant interaction between energetic and thermal particles through the excitation and damping of EGAMs. For instance, if energetic particles excite the main component $m=1$ of EGAM by resonating around the velocity $v_{\|}=v_{\text {res }}$ leading to the EGAM frequency $\omega_{\mathrm{EGAM}}=v_{\text {res }} /(q R)$, the appearance of higher order resonances $m>1$ leads to resonant velocities $v_{\mathrm{res}}^{(m)}=q R \omega_{\mathrm{EGAM}} / m$ with smaller absolute values when $m$ increases and therefore approaching the thermal velocity. In addition, higher order resonances in frequency may occur, leading to frequencies $l \omega_{\mathrm{EGAM}}$, for $l>1$. Therefore, for a given poloidal mode number $m$, this will result in more general expressions for resonant velocities $v_{\mathrm{res}}^{(m, l)}=q R l \omega_{\mathrm{EGAM}} / m$. For the main poloidal harmonic $m=1$, we have $v_{\text {res }}^{(m=1, l)}=$ 
$q R l \omega_{\mathrm{EGAM}}$, representing an interaction between the EGAM and suprathermal particles. In the remainder of this paper, we focus on the nonlinear phase of the EGAM and the formation of the EGAM island. For this purpose we perform three simulations, the only difference being the fraction of energetic particles. For these three simulations we select a D-D plasma and the parameters of the equilibrium distribution functions are $T_{\mathrm{EP}}=2 T_{\mathrm{th}}$ and $v_{\mathrm{EP}}=6 v_{\mathrm{th}}$. The reason for this set of parameters is that the energetic population is sufficiently far from the thermal population and its distribution function sufficiently wide to observe the formation of the EGAM island around the various resonances. The fractions of energetic particles for these three simulations are $n_{\mathrm{EP}}=0.01 n_{\mathrm{eq}}, n_{\mathrm{EP}}=$ $0.02 n_{\mathrm{eq}}$ and $n_{\mathrm{EP}}=0.05 n_{\mathrm{eq}}$. Nevertheless, for the sake of clarity and simplicity, most of the figures will be shown for the intermediate fraction $n_{\mathrm{EP}}=0.02 n_{\mathrm{eq}}$ and the behaviour of the system will be illustrated only for comparison of a few cases. The EGAM frequency has been measured using a Fourier transform of the flux-surface averaged electrostatic potential plotted in figure 2 for the simulation $n_{\mathrm{EP}}=0.02 n_{\mathrm{eq}}$. The Fourier transform of the electrostatic potential over the entire time window is plotted in figure $2 \mathrm{~b}$. The main peak provides the value $\omega_{\mathrm{EGAM}}=4.28 \cdot 10^{-3} \omega_{c, s 0}$. A spectrogram of the time trace of the electrostatic potential (not shown here for the sake of simplicity) would reveal that the mode does not exhibit any chirping. Note that the chirping of EGAM was reported in previous publications, both experimentally [7] and numerically [12]. Very recently, in global gyrokinetic simulations using the ORB5 code [28], a chirping of EGAM was also observed [29], when only wave-particle nonlinearities are kept in the gyro-kinetic equations. It was later shown in [30] that adding wave-wave nonlinearities in that case may result in the strong reduction of the chirping. In our simulations, both wave-particle and wave-wave nonlinearities are kept. This might explain why no chirping is observed for our particular case. In addition, as reported in [31,32], a steady-state solution is not completely excluded, which indicates that a set of parameters exist for which Gysela should not predict any chirping. Nevertheless, further parametric studies and quantitative comparisons between GYSELA and other gyro-kinetic codes such as ORB5 in nonlinear regime are required. This will be done in a future publication, in order to conveniently categorize the behaviour of the EGAM depending on the different parameters that characterize the gyro-kinetic simulations.

The modification of the distribution function is quantified by computing the distance between the equilibrium distribution function and the initial equilibrium distribution function, averaged over time:

$$
\begin{aligned}
\delta F_{\mathrm{eq}}\left(v_{\|}\right)= & \frac{1}{T} \int_{0}^{T} \mathrm{~d} t\left(\left\langle F\left(v_{\|}, \mu=0, \theta, \varphi, t\right)\right\rangle_{\mathrm{FS}}\right. \\
& \left.-F\left(v_{\|}, \mu=0, \theta, \varphi, t=0\right)\right)
\end{aligned}
$$

where $\langle\cdots\rangle_{\text {FS }}$ represents the flux-surface average and $T$ is a sufficiently large time, which is set to the total time of the simulation for the present paper. We first focus only on the value $\mu \approx 0$, i.e. deeply passing particles, since those are not affected by the magnetically trapped particle region, which we will call in the following trapping cone for the sake of simplicity. This perturbed distribution function is plotted for both thermal and energetic particles in figure 3 for the fraction $n_{\mathrm{EP}}=0.02 n_{\mathrm{eq}}$ and represented by thick lines (solid blue line for thermal particles on the left axis and dashed red line for energetic particles on the right axis). The structure, composed of a clump and a hole, is similar to the one obtained in global simulations using OrB5 [33]. Thin vertical lines indicate the position in parallel velocity of the resonances for different values of $l$ and $m$. The main resonance $(l, m)=(1,1)$ is given by dashed black lines. The higher order resonances $m=2$ and $m=3$ for $l=1$ are given by dashed red and blue lines, respectively. The higher order resonance in $l$ for $m=1$ is represented by the dashed-dotted black line. Note that only $l=2$ is visible in the figure. For $l>2$ and $m=1$, the resonant velocity is outside the parallel velocity grid used for the simulation. Finally the higher order resonance in both $m$ and $l$ is shown by the solid magenta line for $(l, m)=(2,3)$. For the sake of clarity all the discussed resonances are labelled in the figure and a magnified view of the $(l, m)=(2,3)$ resonance is provided on the top left panel. Note that higher order resonances satisfying $l=m$ are reduced to the main resonance $(l, m)=(1,1)$. In figure 3 it can be observed that the distribution function of both thermal and energetic particles is perturbed around the various resonances discussed above, showing a good agreement between GYSELA results and nonlinear predictions.

The regions where the distribution function is perturbed have a width that is linked to the width of the island in phase space given by the amplitude of the mode. Since the EGAM is given by $\phi \sim$ $\sin \theta$, observing the EGAM island requires to keep the dependence on the poloidal angle, hence not to perform any flux surface average. Note that avoiding the flux-surface average will not introduce any toroidal dependence, since the EGAM is an axi-symmetric mode. Therefore, the analysis can be performed at a given toroidal angle. Figure 4 shows the perturbed distribution function for thermal (left) and energetic (right) particles as a function of the parallel velocity and the poloidal angle for $\mu \approx 0$, i.e. deeply passing particles, at $\omega_{c, s 0} t=4 \cdot 10^{4}$, which corresponds to a time after nonlinear saturation. The EGAM island at 


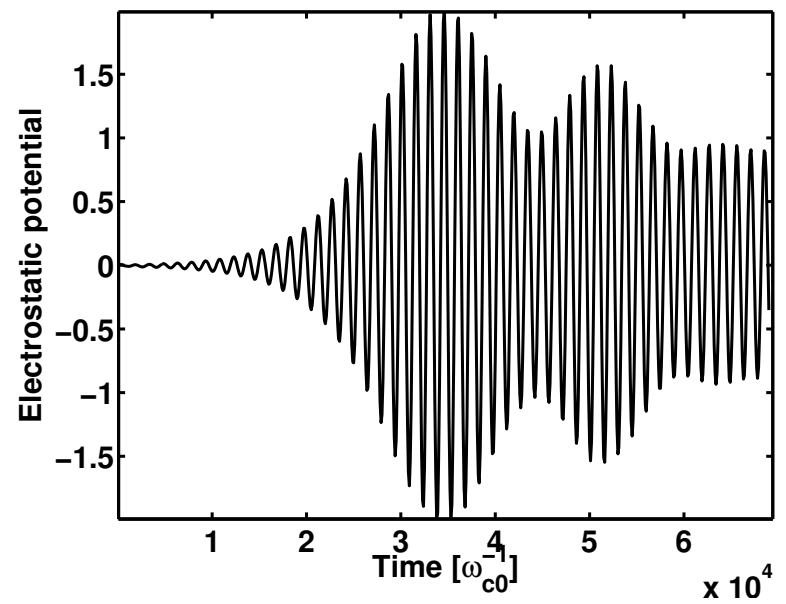

(a)

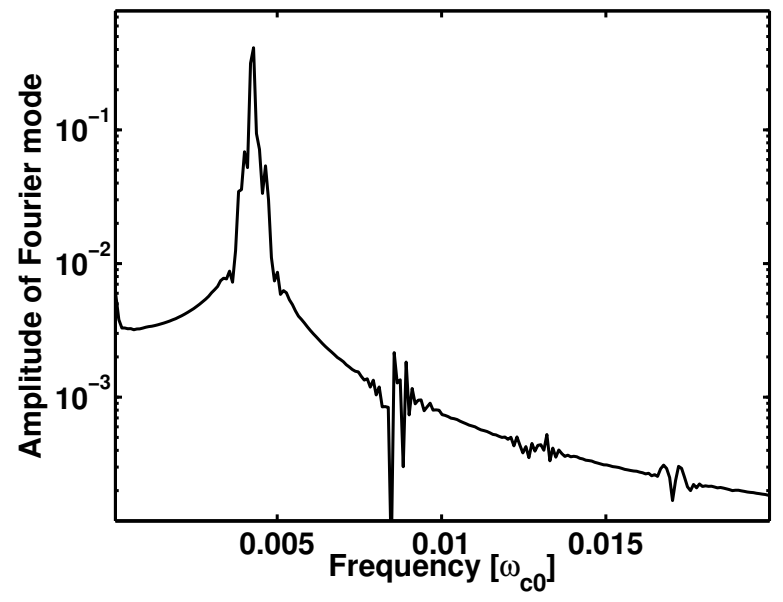

(b)

Figure 2: For the simulation $n_{\mathrm{EP}}=0.02 n_{\mathrm{eq}}$. (Left panel) Time trace of the flux-surface averaged electrostatic potential evaluated at the mid radial position. (Right panel) Fourier transform of the signal plotted on the left panel over the entire time window.

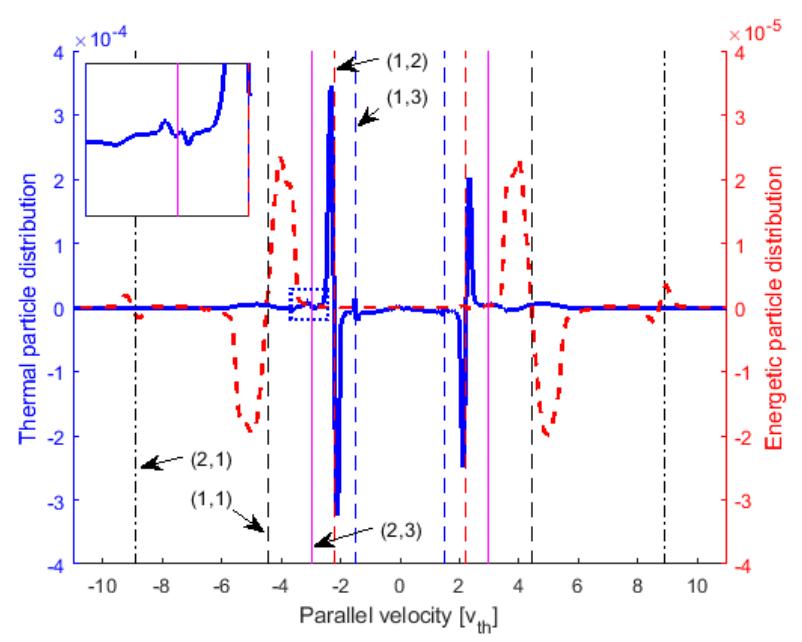

Figure 3: For the simulation with $n_{\mathrm{EP}}=0.02 n_{\mathrm{eq}}$, plotted is the perturbed distribution function as defined by expression 3 for thermal (thick solid blue line) and energetic (thick dashed red line). The vertical thin lines indicate the different resonances $(l, m)$, explained in the text.

the main resonance $(l, m)=(1,1)$ is clearly visible for the energetic particle population, whereas the thermal population is mostly affected by the higher order resonance $(l, m)=(1,2)$ EGAM island. Figure 5 shows the same quantities at the same time but when $\mu$ is increased, so that the presence of the trapping cone can be observed, mainly in the thermal population, which is closer to the trapping cone than energetic population. Moreover further higher order resonances are evidenced for thermal particles, getting closer to the trapping cone and being therefore distorted. When increasing the time well beyond the nonlinear saturation, filamentation can be observed in the phase space. The question that we will address in the following is how the formation of islands in phase space can affect the transport of particles. To perform this analysis in the remainder of the paper we will solve the equations of motion for test particles and determine the existence of chaotic region in phase space responsible for the transport of particles. It is though to be noted that the analysis of the particle transport that will be performed in the next sections should be ideally carried out using a self-consistent approach, i.e. with particles that have an impact on the electrostatic potential. Nevertheless, the goal of this work is to explain the fundamental mechanism for the losses due to axisymmetric modes and the selfconsistent approach is therefore beyond the scope of the present manuscript and left for future publications.

\section{Integration of the equations of motion of gyro-centres in toroidal geometry}

Within the electrostatic limit, the evolution of the gyro-centre coordinates of species $s$ solved in GYSELA code is given by the equations [26]

$$
\begin{aligned}
& \frac{\mathrm{d} x^{i}}{\mathrm{~d} t}=v_{\|} \mathbf{b}_{s}^{*} \cdot \nabla x^{i}+\mathbf{v}_{E} \cdot \nabla x^{i}+\mathbf{v}_{D} \cdot \nabla x^{i} \\
& m_{s} \frac{\mathrm{d} v_{\|}}{\mathrm{d} t}=-\mu \mathbf{b}_{s}^{*} \cdot \nabla B
\end{aligned}
$$




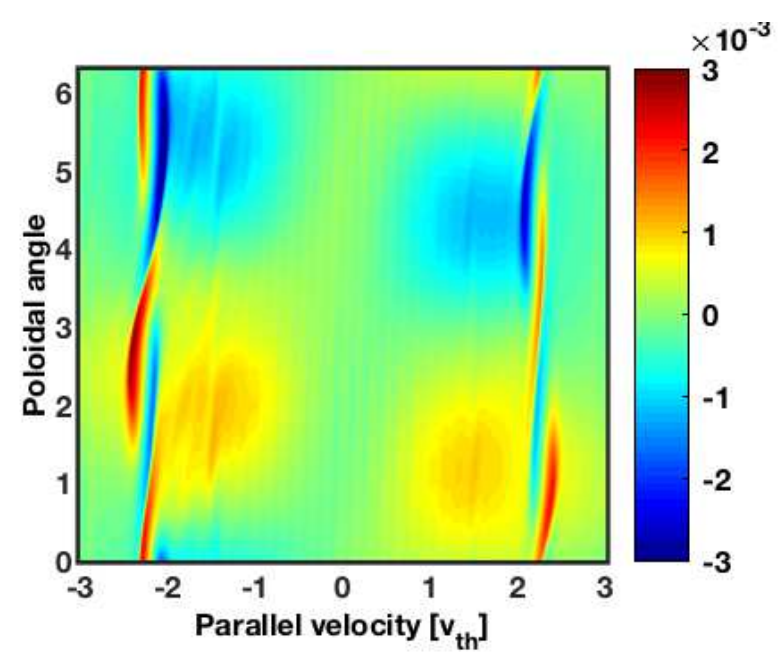

(a)

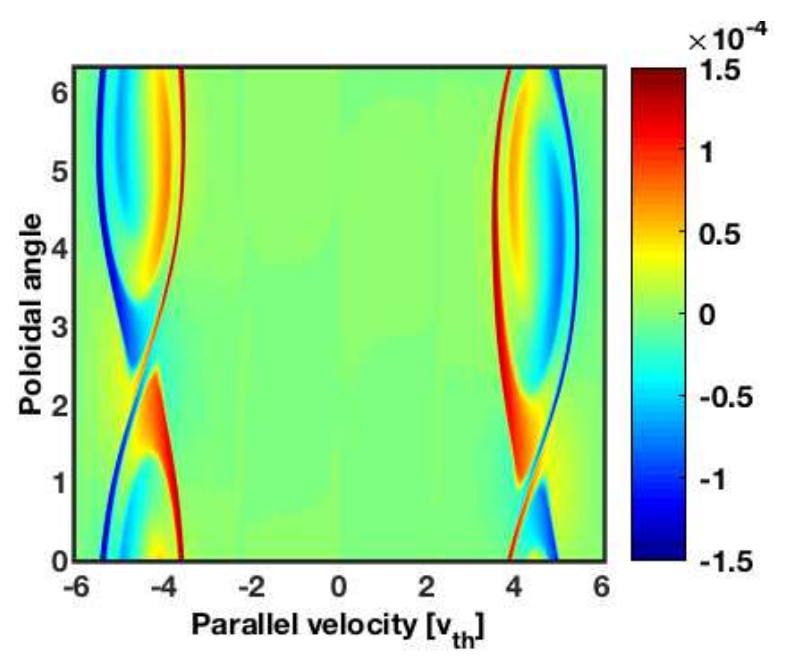

(b)

Figure 4: Snapshots of the perturbed distribution function for the simulation $n_{\mathrm{EP}}=0.02 n_{\mathrm{eq}}$ for thermal (left panel) and energetic (right panel) particles as a function of the parallel velocity and the poloidal angle, for $\mu \approx 0$.

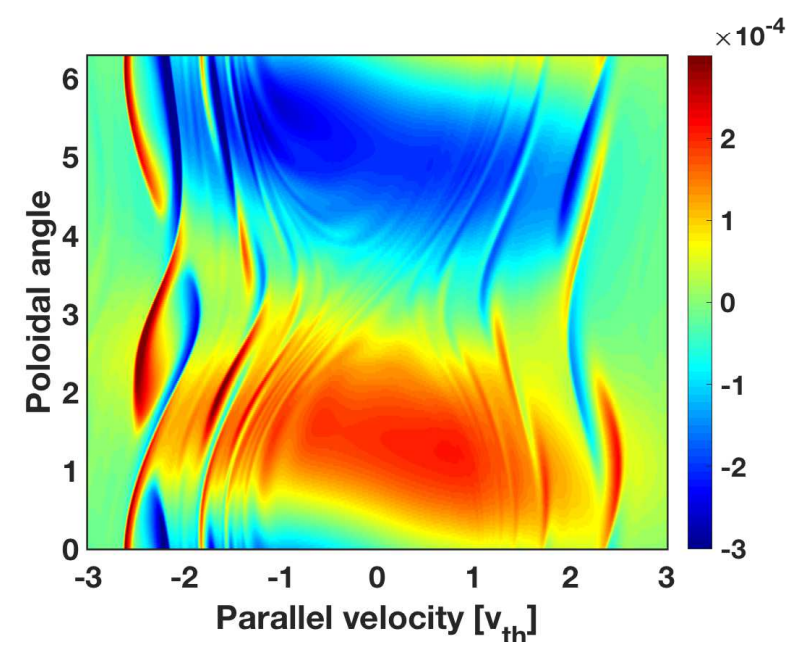

(a)

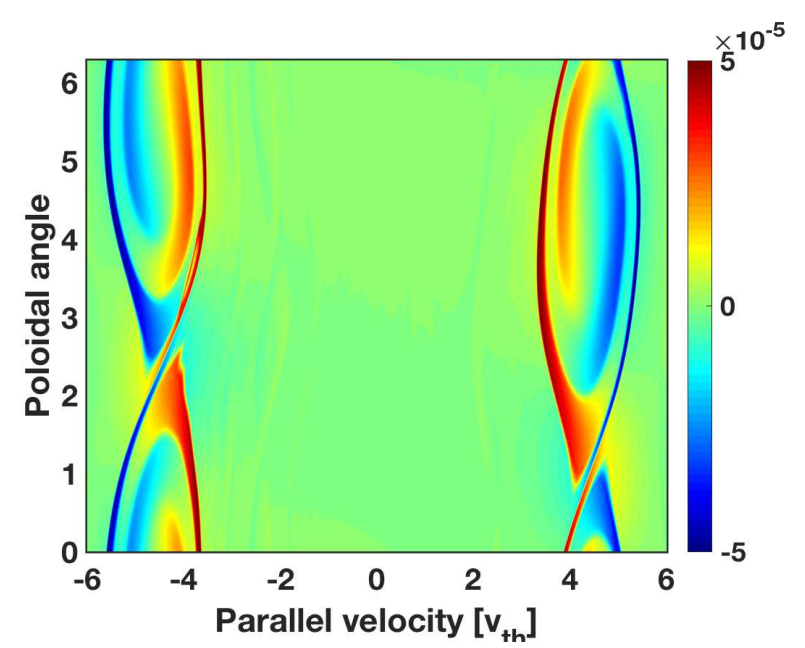

(b)

Figure 5: Snapshots of the perturbed distribution function for the simulation $n_{\mathrm{EP}}=0.02 n_{\mathrm{eq}}$ for thermal (left panel) and energetic (right panel) particles as a function of the parallel velocity and the poloidal angle, for $\mu=6$, normalized to $B_{0} / T_{0}$.

$$
-e Z_{s} \mathbf{b}_{s}^{*} \cdot \nabla J_{0} \phi+\frac{m_{s} v_{\|}}{B} \mathbf{v}_{E} \cdot \nabla B
$$

where $x^{i}$ is the $i$ th contravariant component of the coordinate $\mathbf{x}\left(x^{1} \equiv r\right.$ in the radial direction, $x^{2} \equiv \theta$ in the poloidal direction and $x^{3} \equiv \varphi$ in the toroidal direction), $v_{\|}$the parallel component of the velocity along the magnetic field lines, $\mathbf{v}_{E}$ is the $\mathbf{E} \times \mathbf{B}$ drift, $\mathbf{v}_{D}$ is the magnetic drift, $\mu$ is the magnetic moment, which is an invariant within the present model, $m_{s}$ is the mass of particles, $e$ is the elementary charge, $Z_{s}$ is the atomic number, $\phi$ is the electrostatic potential, $B$ is the magnitude of the magnetic field, $J_{0}$ is the gyro-average operator and $\mathbf{b}_{s}^{*}$ is defined as

$\mathbf{b}_{s}^{*}=\frac{\mathbf{B}}{B_{\|, s}^{*}}+\frac{m_{s} v_{\|}}{e Z_{s} B_{\|, s}^{*} B} \nabla \times \mathbf{B}$

with

$B_{\|, s}^{*}=B+\frac{m_{s}}{e Z_{s}} v_{\|} \mathbf{b} \cdot \nabla \times \mathbf{b}$

the volume element in guiding-centre velocity space, 
where we have introduced the magnetic field $\mathbf{B}$ and the unit vector along the magnetic field lines $\mathbf{b}$. The fundamentals of tensor calculus and toroidal geometry used in Gysela code and in this paper are summarized in appendix Appendix A. In particular, we use the simple model of concentric circular magnetic flux surfaces, with the magnetic field defined by equation A.18. With this simple model, we show in appendix Appendix A that the radial and poloidal contravariant components of $\mathbf{b}_{s}^{*}$ vanish and that the only contravariant component of $\mathbf{b}_{s}^{*}$ that remains is therefore the toroidal one.

The $i$ th contravariant components of the drifts are calculated as follows

$\mathbf{v}_{E} \cdot \nabla x^{i}=\frac{1}{B_{\|, s}^{*}} \mathbf{b} \cdot\left(\nabla J_{0} \phi \times \nabla x^{i}\right)$

$\mathbf{v}_{D} \cdot \nabla x^{i}=\frac{m_{s} v_{\|}^{2}+\mu B}{e Z_{s} B_{\|, s}^{*} B} \mathbf{b} \cdot\left(\nabla B \times \nabla x^{i}\right)$

where the triple product is calculated for two scalar fields $F$ and $G$ as

$\mathbf{b} \cdot(\nabla F \times \nabla G)=\frac{1}{\mathcal{J}_{x}} \epsilon^{i j k} b_{k} \partial_{i} F \partial_{j} G$

with $\mathcal{J}_{x}=r R$ the Jacobian in space and $\epsilon^{i j k}$ the Levi-Civita tensor. The covariant and contravariant components of a vector $\mathbf{A}$ are calculated as explained in appendix Appendix A.

For convenience, when comparing with Gysela simulations we use the same normalisations with respect to a reference species $s 0$ as in the code, i.e. the time is normalized to the cyclotron period of the reference species $t=\hat{t} / \omega_{c, s 0}$, all the distances are normalized to the thermal Larmor radius of the reference species $L=\hat{L} \rho_{s 0}$, the mass and charge of species $s$ are trivially normalized as $m_{s}=\hat{A}_{s} m_{s 0}$ and $e Z_{s}=\hat{Z}_{s} Z_{s 0}$, respectively. However, parallel and perpendicular velocities of species $s$ are normalized to the thermal velocity of the species $s$ itself, i.e. $v_{\|}=v_{\mathrm{th}, s} \hat{v}_{\|}$and $v_{\perp}=v_{\mathrm{th}, s} \hat{v}_{\perp}$. The magnetic field is normalized to the value of the magnetic field at the magnetic axis, i.e. $B=\hat{B} B_{0}$ and the electrostatic potential is normalized as $\phi=e \hat{\phi} / T_{0}$, where $T_{0}$ is the reference electron temperature. In the previous expressions, the $\hat{i}$ symbol represents normalized quantities. With these normalisations, the normalized equations of motion of gyro-centres read

$$
\begin{aligned}
\frac{\mathrm{d} x^{i}}{\mathrm{~d} t}= & \frac{1}{\sqrt{A_{s}}} v_{\|} \mathbf{b}_{s}^{*} \cdot \nabla x^{i}+\mathbf{v}_{E} \cdot \nabla x^{i}+\mathbf{v}_{D} \cdot \nabla x^{i} \\
\frac{\mathrm{d} v_{\|}}{\mathrm{d} t}= & -\frac{1}{\sqrt{A_{s}}} \mu \mathbf{b}_{s}^{*} \cdot \nabla B \\
& -\frac{Z_{s}}{\sqrt{A_{s}}} \mathbf{b}_{s}^{*} \cdot \nabla J_{0} \phi+\frac{v_{\|}}{B} \mathbf{v}_{E} \cdot \nabla B
\end{aligned}
$$

where all quantities are normalized, the $\hat{\text { s symbols have }}$ been dropped for the sake of clarity and expression 5 is rewritten as

$\mathbf{b}_{s}^{*}=\frac{\mathbf{B}}{B_{\|, s}^{*}}+\frac{\sqrt{A_{s}} v_{\|}}{Z_{s} B_{\|, s}^{*} B} \nabla \times \mathbf{B}$

with

$B_{\|, s}^{*}=B+\frac{\sqrt{A_{s}}}{Z_{s}} v_{\|} \mathbf{b} \cdot \nabla \times \mathbf{b}$

To make further progress we need to explicitly calculate $B_{\|, s}^{*}$ and $\mathbf{b}_{s}^{*}$, the contravariant components of the drift velocities and the projection of the $\nabla$ operator on $\mathbf{b}_{s}^{*}$. These details of the calculation can be found in Appendix Appendix B. The final set of equations of motion is

$$
\begin{aligned}
\alpha \frac{\mathrm{d} r}{\mathrm{~d} t}= & -\frac{1}{B} \frac{1}{r} \frac{\partial \bar{\phi}}{\partial \theta}-\frac{v_{\|}^{2}+\mu B}{Z_{s} B^{2}} \frac{1}{r} \frac{\partial B}{\partial \theta} \\
\alpha \frac{\mathrm{d} \theta}{\mathrm{d} t}= & \frac{1}{\sqrt{A_{s}}} \frac{1}{q R_{0}} v_{\|}+\frac{1}{B} \frac{1}{r} \frac{\partial \bar{\phi}}{\partial r}+\frac{v_{\|}^{2}+\mu B}{Z_{s} B^{2}} \frac{1}{r} \frac{\partial B}{\partial r} \\
\alpha \frac{\mathrm{d} \varphi}{\mathrm{d} t}= & \frac{1}{\sqrt{A_{s}}} \frac{1}{R_{0}} v_{\|}+\alpha \frac{v_{\|}^{2}}{Z_{s} B_{\|, s}^{*} B} \frac{1}{r R} \frac{\partial}{\partial r}\left(\frac{r^{2}}{q R}\right) \\
\alpha \frac{\mathrm{d} v_{\|}}{\mathrm{d} t}= & -\frac{\mu}{\sqrt{A_{s}}} \frac{1}{q R_{0}} \frac{\partial B}{\partial \theta}-\frac{Z_{s}}{\sqrt{A_{s}}} \frac{1}{q R_{0}} \frac{\partial \bar{\phi}}{\partial \theta} \\
& +\frac{v_{\|}}{B^{2}} \frac{1}{r}\left(\frac{\partial \bar{\phi}}{\partial r} \frac{\partial B}{\partial \theta}-\frac{\partial \bar{\phi}}{\partial \theta} \frac{\partial B}{\partial r}\right)
\end{aligned}
$$

where $\alpha=B_{\|, s}^{*} / B \sqrt{1+\zeta^{2}}, \zeta=r /\left(q R_{0}\right), q$ being the safety factor and $R_{0}$ the major radius at the magnetic axis. The time integration of these equations is done with a 4th order Runge-Kutta explicit algorithm. The interpolation of the fields when following the test particles can be done by using an Inverse-DistanceWeighting (IDW) method with a power parameter $p>$ 1 for 2D interpolations [34] or Lagrange polynomials on 4 or 16 points in the poloidal cross section. The diagnostics are saved from the simulations every two time steps. For the post-treatment analysis the same time step as in the simulations is used, which means that we need to interpolate in time the electrostatic potential in between two diagnostics. For this we use linear interpolation.

As an example of the test-particle tracking posttreatment, we show in the top panel of figure 6 the projection onto the poloidal cross-section of the trajectories of two particles, passing (left) and trapped (right), with a total energy $E=14 E_{\mathrm{th}}$. The difference between the passing and the trapped particle is the pitch angle defined as $\Lambda=\mu B_{0} / E$. For the passing particle $\Lambda=0.43$, whereas for the trapped particle $\Lambda=0.86$. The external circle represents the boundary of the simulation. The set of equations $12 a-12 d$ conserves energy and toroidal momentum (see appendix Appendix C). The time traces of the relative error of the energy (thick black line) and 


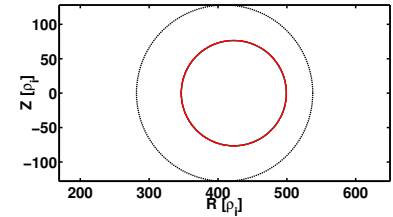

(a)

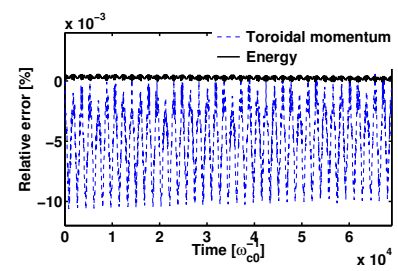

(c)

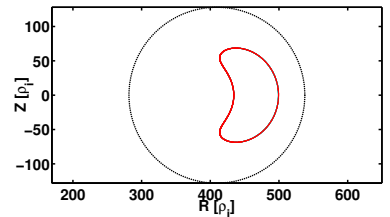

(b)

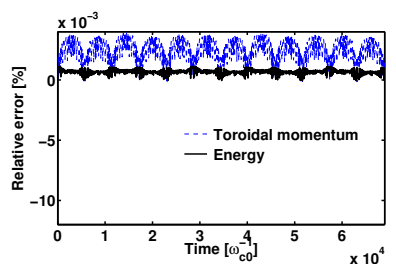

(d)

Figure 6: Top panel: poloidal projection of the unperturbed trajectories of a passing (left) and a trapped (right) particle having the same energy. Bottom panel: relative error of toroidal canonical momentum (thin blue line) and energy (thick black line) for passing (left) and trapped (right) particles.

the toroidal canonical momentum (thin blue line) for both passing (left) and trapped (right) particles are plotted in the bottom panel of figure 6 . In terms of conservation properties, Lagrange interpolation is preferred to IDW method, since the latter increases the relative error by two orders of magnitude. For the Lagrange interpolation, regarding the number of points, it has been observed that 4 points Lagrange interpolation is enough to achieve a relative error up to $\sim 0.0001 \%$ for the energy and $\sim 0.01 \%$ for the toroidal canonical momentum.

\section{Losses of particles due to EGAM}

In the present section we use the particle tracking posttreatment explained earlier and apply it to an ensemble of particles within $0<\mu<16$ and $-7<v_{\|}<7$ at two different radial positions representing an inner region $(r / a=0.2)$ and an outer region $(r / a=0.6)$. For the remainder of the paper, in order to analyse the radial transport of gyro-centres, we run GYSELA simulations with a larger radial domain, which translates into a smaller normalized thermal ion Larmor radius $\rho_{\star}=$ 1/150. All particles are initialized at $\theta=0$ and $\varphi=0$. The equations of motion are solved for the three energetic particle fractions: $n_{\mathrm{EP}}=0.01 n_{\mathrm{eq}}$, $n_{\mathrm{EP}}=0.02 n_{\mathrm{eq}}$ and $n_{\mathrm{EP}}=0.05 n_{\mathrm{eq}}$. The result is summarized in figure 7. Losses represented by blue points indicate prompt losses. These are losses that exist even in the absence of EGAMs due to the fact

that the initialisation of particles is such that their orbits intercept the boundary and are lost from the very beginning of the simulation. These losses are also called first-orbit prompt losses. Red points represent losses that exist only in the presence of EGAMs and are therefore called EGAM losses. Several comments can be made based on the observation of this figure. First, mainly counter-passing particles experience EGAM losses, independently of the radial position and the energetic particle fraction. Second, the region of initial conditions where particles are lost is denser when increasing the energetic particle fraction. Actually, for small fraction of energetic particles (top panel of figure 7), EGAM losses occur only for outer radial positions. This indicates that EGAMs have indeed an impact on the transport and losses of particles. The transport of particles in phase space due to the EGAM might modify the orbits in the sense that passing particles might be trapped and trapped particles might be de-trapped. This is due to the conservation of the toroidal momentum $P_{\varphi}=-Z_{s} \psi+\sqrt{A_{s}} \frac{B_{\varphi}}{B} v_{\|}$, which makes the link between the radial position given by the poloidal flux $\psi$ and the parallel velocity $v_{\|}$. For ions $\left(Z_{s}>0\right)$, conserving $P_{\varphi}$ implies that an acceleration of particles leads to a radial transport towards the outer region of the tokamak. The acceleration in the parallel direction makes counter-passing particles approach the trapping-cone and become trapped. Their orbits can subsequently intercept the wall of the tokamak, as speculated in experimental work [7] and confirmed in simulations [8]. Note that for co-passing particles that approach the trapping-cone, their parallel velocity is decreased. Therefore the radial position is also reduced owing to the conservation of $P_{\varphi}$. They are therefore transported to a region where the trapping-cone is narrower. As a result co-passing particles are unlikely trapped by the magnetic field. This explains why neither the trapping/de-trapping mechanism due to EGAM nor the losses shown in figure 7 are symmetric with respect to $v_{\|}$. This asymmetry might shed some light on the preferential energetic particle injection direction. In particular, co-injection of energetic particles would likely reduce the losses due to EGAMs. More in depth analysis of the EGAM losses indicates that the red points in figure 7 are actually two types of losses. To clarify this, we have analysed the exit time of particles, $t_{\text {exit }}$, defined as the time a particle takes to reach the boundary of the simulation. We have subsequently obtained the histogram of the exit time for two radial positions $(\mathrm{r} / \mathrm{a}=0.2$ and $\mathrm{r} / \mathrm{a}=0.6)$ for the simulation with $n_{\mathrm{EP}} / n_{\mathrm{eq}}=0.02$. This is given in figure 8. The blue lines indicate the fuirst-orbit prompt losses and the red lines the EGAM-indeuced losses. The exit time is normalized to the saturation time of the EGAM, $t_{\mathrm{sat}, \mathrm{EGAM}}$. The saturation time is measured by 
locating the global maximum of the time trace of the electrostatic potential. Therefore, $t_{\mathrm{sat}, \mathrm{EGAM}} / t_{\text {exit }}>$ 1 represents events that occur before the EGAM saturation, where linear effects are dominant, and $t_{\text {sat,EGAM }} / t_{\text {exit }}<1$ represents events that occur after the EGAM saturation, where nonlinear effects are dominant. The separation between both domains is highlighted by a vertical line. It is evidenced that prompt losses occur well before the EGAM is excited, which indicates that these losses are independent of the EGAM, as expected. However, the EGAM losses can be divided into losses occurring before and after the EGAM saturation. One can see that for particles initialized in the inner region of the tokamak, losses are mainly due to the nonlinearities, whereas for particles initialized in the outer region of the tokamak a significant fraction of losses occurs during the linear phase. In the left frame, we show the projection onto the poloidal cross-section of two particles that are lost during the linear excitation of the EGAM. We have identified two classes of particles: counter-passing particles that are sufficiently close to the trapping cone (left) and trapped particles that approach the boundary when $v_{\|}>0$. For those particles the transfer of energy during the linear phase is enough to induce their losses after very few periods. These losses are therefore called EGAM-enhanced prompt losses. Note that these losses are similar to the losses reported in $[35,36]$, but in the electrostatic limit. In the following we analyse in detail the dynamics of the particles initialized in the inner region of the tokamak and lost during the nonlinear regime and we provide an explanation for the fundamental underlying mechanism of the transport and losses.

The losses reported here imply that a transport due to EGAMs is possible. To elucidate whether this transport is due to the interaction between the mode and the particles in velocity space or not, we analyse the trajectories of individual particles. If an interaction in velocity space occurs, the conservation of $P_{\varphi}$ will lead to a radial transport of particles and eventually their losses. The trajectories of three particles identified as nonlinearly-EGAM-induced lost particles are studied in detail and plotted in the top panel of figure 9. The left panel shows the projection onto the poloidal cross-section of one of the particle trajectories represented by solid blue lines (note that for the sake of clarity, only one trajectory can be plotted on the cross-section). The middle panel shows the time trace of the parallel velocity for the three particles (solid blue line, dashed black line and dotted red line), and the radial position of the particles as a function of time is given in the right panel. For reference, the bottom panel shows the same quantities for the unperturbed trajectory of the blue particle, i.e. the trajectory of the blue particle without EGAM. For both perturbed and unperturbed trajectories, the boundary domain in real space, corresponding to a circular flux-surface, is given in the left panel and represented by a dotted black line. The three particles are initialized with slightly different parallel velocities in the neighbourhood of $v_{\|}(t=0)=-5.5 v_{\mathrm{th}}$, which is close to the main EGAM resonance, and in figure 3 it is located on the left side of the resonance indicated by the black dashed line. The three particles are also initialized with slightly different magnetic moments in the neighbourhood of $\mu=13 T_{0} / B_{0}$. When the EGAM is excited, the particle parallel velocities start exhibiting low frequency oscillations during a syncrhonisation process with the $m=1$ component of the mode. This phase is represented by the magnified view only for the blue particles. Depending on the analysed particle, the subsequent behaviour exhibits different characteristics. For example, for the blue particles, these oscillations end at the same time as the EGAM saturates $(t \approx 35000)$. At that moment the particle experiences a motion that makes it cross the main EGAM island (it is de-trapped from the EGAM) and $v_{\|}$explores the range between the harmonic $m=$ 1 (indicated by a horizontal dashed black line) and $m=2$ (indicated by a horizontal dashed red line). The proximity between the $m=2$ harmonic and the trapping-cone makes the particle become trapped and eventually lost. However, the black particle remains trapped inside the EGAM longer and only around $t \approx 80000$ is it de-trapped, approaching the trappingcone and being eventually lost. Finally, the red particle exhibits a trapping-detrapping (inside EGAM) oscillating state in an apparently random manner until it interacts with the trapping-cone and is eventually lost. This particle takes much more time to be lost than the particle corresponding to the black and blue lines.

To elucidate the reason for this behaviour, we perform the same test-particle tracking for an ensemble of particles initialized close to the main EGAM resonant velocity for the simulation $n_{\mathrm{EP}}=0.02 n_{\mathrm{eq}}$. We carry out this analysis only for particles initialized at $r / a=0.2$, i.e. well confined into the inner core of the tokamak. Since the main losses in that region come from particles having a high $\mu$ value, we initialize particles within the interval $\mathcal{S}_{\mu}=\{12<\mu<16\}$. The parallel velocity is initialized within the interval $\mathcal{S}_{v_{\|}}=\left\{-6<v_{\|}<-5\right\}$. The ensemble of tracers is defined using a uniform distribution of particles in both domains. Note that, since the distribution function we use in the present paper to excite EGAMs is $F_{\text {eq }} \propto \exp (-\mu B)$, the fraction of particles in $\mathcal{S} \times \mathcal{S}_{v_{\|}}$ is $\approx 2 \cdot 10^{-4 \%}$ of the total energetic particle population. Nevertheless, it was shown in [9] that EGAMs can be 


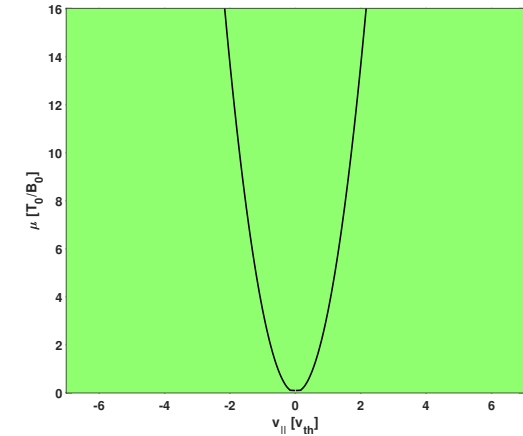

(a)

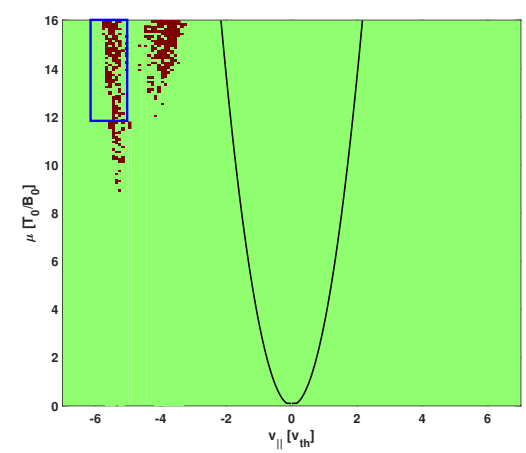

(c)

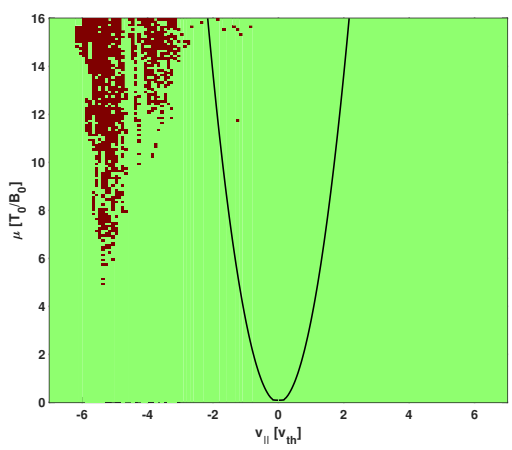

(e)

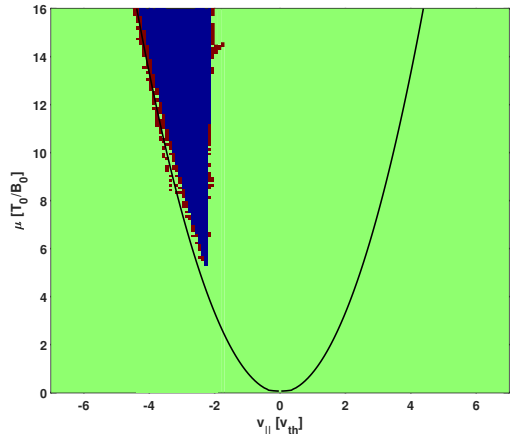

(b)

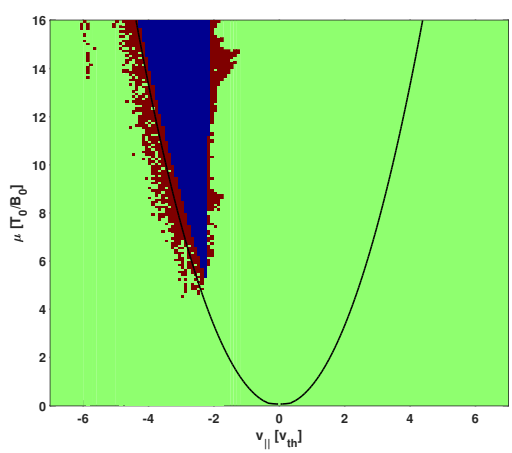

(d)

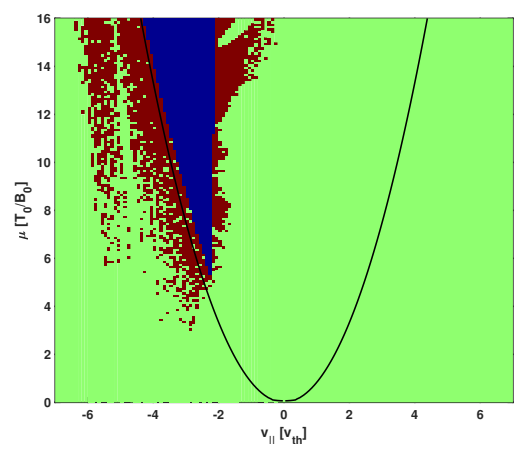

(f)

Figure 7: Losses of particles for three fractions of energetic particles, $n_{\mathrm{EP}}=0.01 n_{\mathrm{eq}}$ (top panel), $n_{\mathrm{EP}}=0.02 n_{\mathrm{eq}}$ (middle panel) and $n_{\mathrm{EP}}=0.05 n_{\mathrm{eq}}$ (bottom panel) for particles initialized at $r / a=0.2$ (left) and $r / a=0.6$ (right). The black line represents the trapping region. Blue (resp. red) points represent prompt (resp. EGAM) losses. 


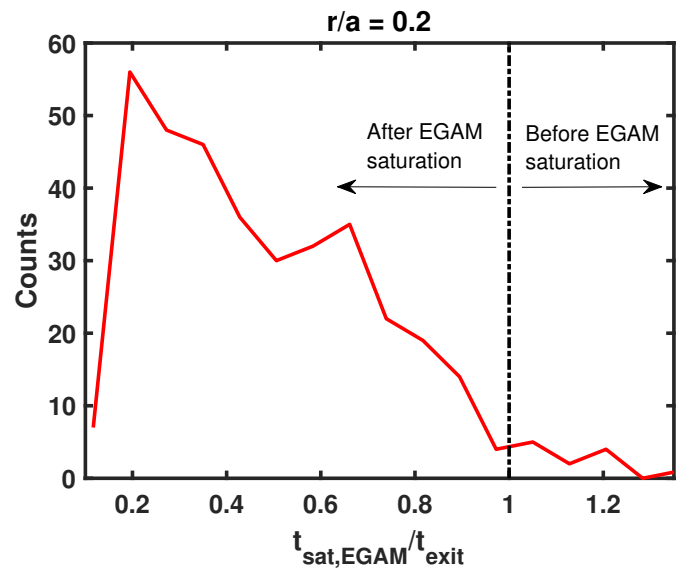

(a)

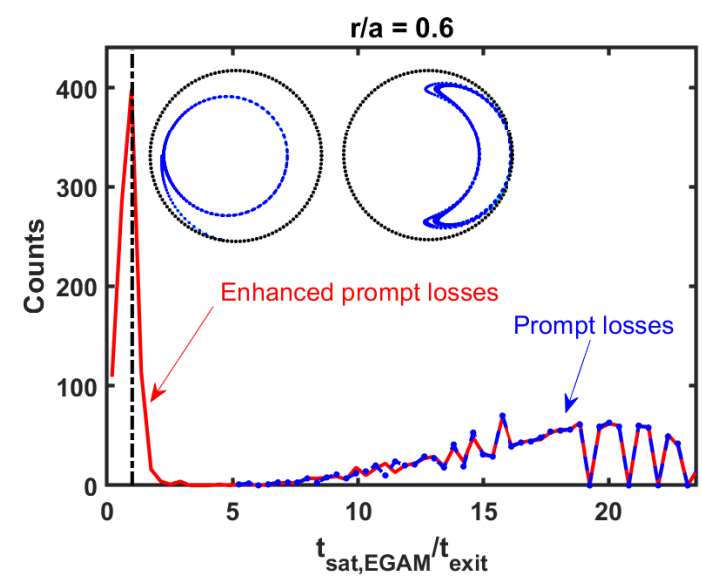

(b)

Figure 8: Histogram of the exit time of particles for the simulation $n_{\mathrm{EP}}=0.02 n_{\mathrm{eq}}$ at two radial positions $r / a=0.2$ (left) and $r / a=0.6$ (right). In the right panel we plot also the projection onto the poloidal cross-section of two EGAM-induced lost particles before the saturation of the mode.
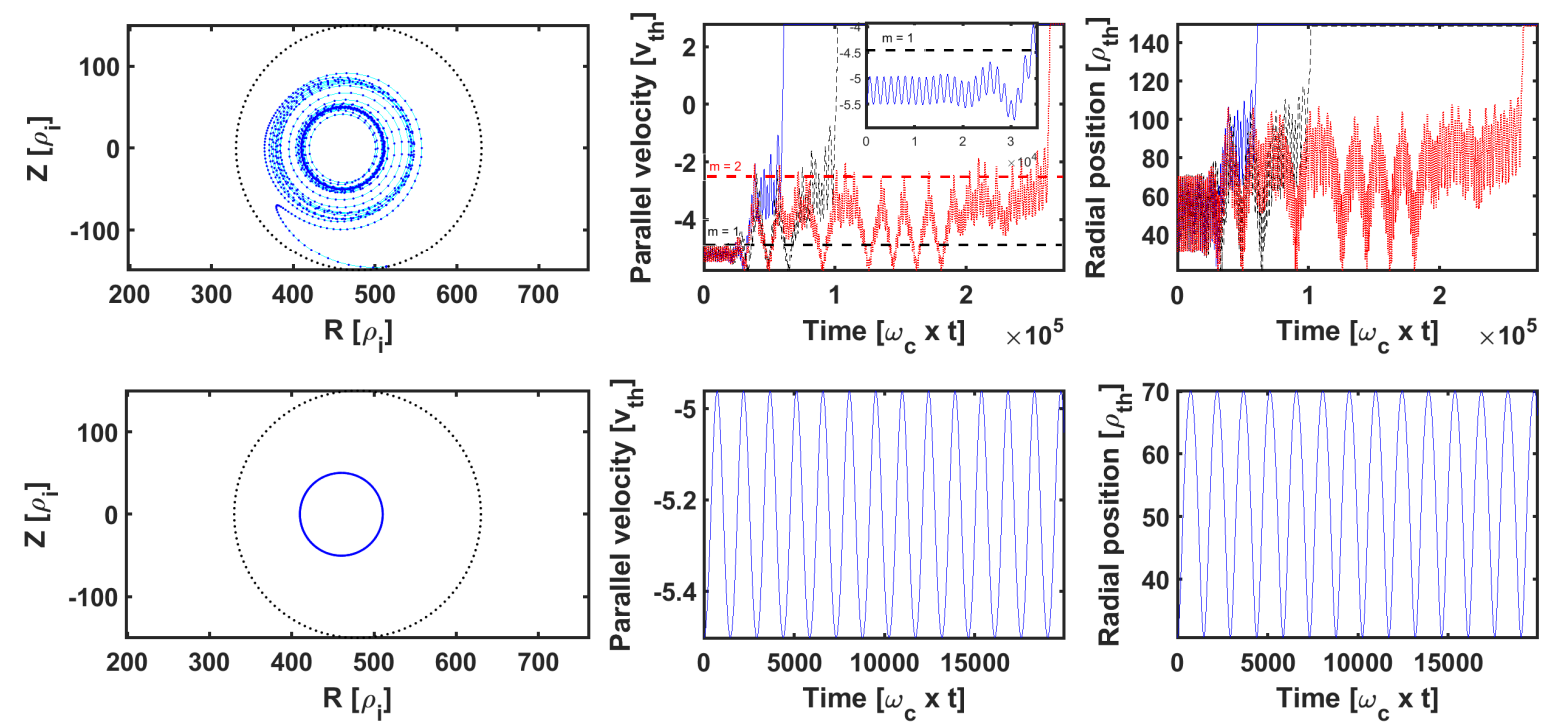

Figure 9: Perturbed (top) and unperturbed (bottom) trajectories of test gyro-centres. The middle and right panels on the top present trajectories of three particles that are lost at different times during the simulation. The left panel on the top presents only the projection onto the poloidal cross section for one of the particles (dotted blue line). For both perturbed and unperturbed trajectories, the boundary domain in real space, corresponding to a circular flux-surface, is given in the left panel and represented by a dotted black line. 
excited in Gysela with a more experiment-relevant equilibrium distribution function. This function was built upon a slowing-down distribution exhibiting a strong dependence on the pitch angle [4] and the properties of the EGAMs were found to be similar independently of the distribution function. We can for instance consider a critical energy $E_{c} \approx 14 E_{\text {th }}$ assume that energetic particles are born with an energy $E_{b} \approx 40 E_{\text {th }}$ and a pitch angle $\Lambda_{0}=0.5$. We also consider that the distribution in pitch angle has a width $\Delta \Lambda=0.2$. With these parameters, the fraction of energetic particles in the interval $\mathcal{S}_{v_{\|}}$would be $\approx 8 \%$ of the total energetic particle population and the pressure associated with these particles would represent $\approx 9 \%$ of the energetic particle pressure.

Since the main dynamics of the EGAM occurs in the $\left(\theta, v_{\|}\right)$sub-space, we use a higher resolution in $v_{\|}$ than in $\mu$. In order to improve the statistics of our analysis, the total number of integrated trajectories is $\sim 16000$. This domain is indicated in the left-hand side top panel of figure 7 by a blue solid rectangle. The integration is done by using 16 points in the poloidal cross-section for interpolation and one additional point in between two Gysela diagnostics. The losses of particles in $\left(v_{\|}, \mu\right)$ space are represented in the left panel of figure 10. The losses diagram exhibits a cloud of points aligned with the trapping cone. This indicates that the interaction between the EGAM and the trapping cone might indeed be a key point for the dynamics of the physical system. Nevertheless, no clear pattern can be identified within the cloud of points, suggesting that a deterministic process is unlikely to result in the observed losses. The number of particles that are lost is plotted as a function of time in the middle panel of figure 10, represented by a black line. The time-trace of the flux-surface averaged electrostatic potential is over-plotted and represented by a magenta line. This figure indicates that there is a maximum in the number of losses right after the EGAM saturates and the losses occur during the whole nonlinear phase of the EGAM, suggesting that the losses are due to nonlinear effects. Finally, the time-Fourier transform of the losses of particles after filtering out the zero frequency has been performed and plotted in the right panel of figure 10. The amplitude of the Fourier modes exhibit dominant components at the EGAM frequency and its harmonics, in agreement with experimental results [7].

To explain why the losses occur at the EGAM frequency and only during the nonlinear phase we plot in figure 11 the position of the whole ensemble of particles, i.e. for all $\mu \in \mathcal{S}_{\mu}$, in the $\left(v_{\|}, \theta\right)$ subspace at different instants, indicated by dashed vertical lines (blue, red and green, respectively) in the middle panel of figure 10: (1) before, (2) just after and (3) well after the nonlinear saturation of the EGAM. For the sake of clarity, the radial position of particles is indicated by colours, being blue and red the two limits representing $r / a \rightarrow 0$ and $r / a \rightarrow 1$, respectively. The trapping cone is represented by a dashed black line. This region is calculated as follows. For each point in the poloidal angle grid of GYSELA, $\theta_{g} \in[0,2 \pi[$, we determine the ensemble $\mathcal{S}_{\theta_{g}}$ of test particles with a poloidal angle between $\theta_{g}$ and $\theta_{g}+\Delta \theta_{g}$, where $\Delta \theta_{g}$ is the grid step in the poloidal direction. Since the trapped fraction increases with the minor radius, we subsequently identify the particle at the outest radial position, i.e. we determine $i$ such that $r_{i}=$ $\max _{j \in \mathcal{S}_{\theta_{g}}} r_{j}$. Once this particle is identified by its radial position $r_{i}$, its poloidal angle $\theta_{i} \in\left[\theta_{g}, \theta_{g}+\Delta \theta_{g}\right]$ and its magnetic moment $\mu_{i}$, we calculate the parallel velocity $v_{\|, \text {sep }}$ the particle would have if it was on the separatrix of the unperturbed trapping cone. For this purpose we make use of the conservation of the energy, which establishes that the energy at the $\mathrm{X}$-point must equal the energy at any point of the separatrix, and assume that the radial position $r_{i}$ of the particle is the same as the position of the particle on the separatrix. Note that the conservation of energy is not satisfied in our physical system, since the Hamiltonian depends explicitly on time. Therefore, the trapping cone we obtain must be understood as an unperturbed trapping cone. Under these assumptions, the only equation of the trapping cone is

$\mu_{i} \frac{\sqrt{1+\left(\frac{r_{i}}{q R_{0}}\right)^{2}}}{1-\frac{r_{i}}{R_{0}}}=\frac{1}{2} v_{\|, \mathrm{sep}}^{2}+\mu_{i} \frac{\sqrt{1+\left(\frac{r_{i}}{q R_{0}}\right)^{2}}}{1+\frac{r_{i}}{R_{0}} \cos \theta_{i}}$

where all quantities are normalized as in Gysela. In the left panel of figure 11 it is observed that some particles remain passing (in terms of EGAM island) and other particles are trapped inside the EGAM. The trapping of some of the particles allows one to observe clearly the formation of the EGAM island in phase space. In addition, as stated earlier in this paper, the main EGAM island is distorted by the presence of the trapping-cone located around $v_{\|}=0$. In the same panel, the initial ensemble of tracers is represented by a solid black line. When the EGAM saturates, the EGAM island strongly interacts with the trappingcone, as observed in the middle panel of figure 11 . This panel shows the instant where the first particles of the ensemble are lost and indicates that the losses are produced by the interaction between the O-point of the EGAM island and the $\mathrm{X}$-point of the trapping cone. The right panel of figure 11 shows the same structure later on in the simulation, where a chaotic channel from counter-passing particles and lost-particles is observed through the interaction of the EGAM island and the $\mathrm{X}$-point of the trapping-cone. This interaction occurs 


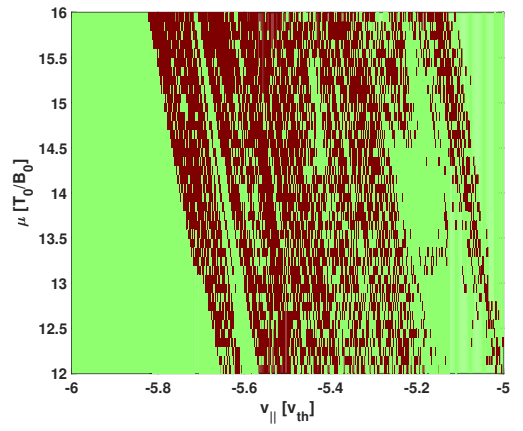

(a)

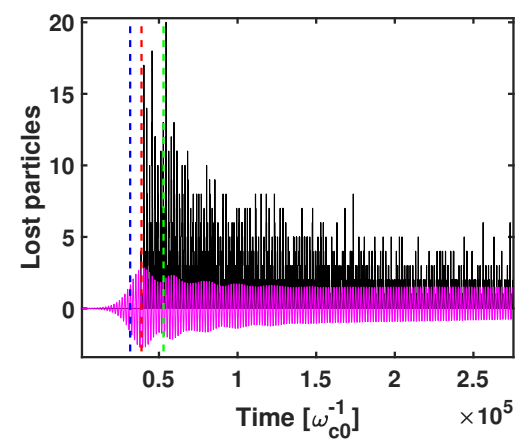

(b)

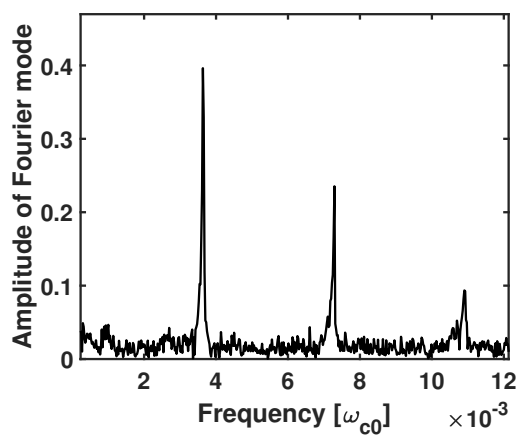

(c)

Figure 10: (Left) Losses of particles for the zoomed area shown in figure 7. (Middle) Time trace of the losses of particles (black line) together with the time-trace of the electrostatic potential (magenta line). (Right) Fourier transform of the time-trace of the losses of particles, exhibiting dominant components at the EGAM frequency and its harmonics.

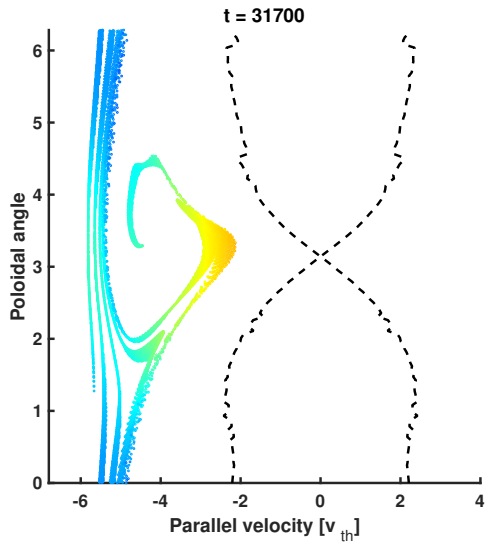

(a)

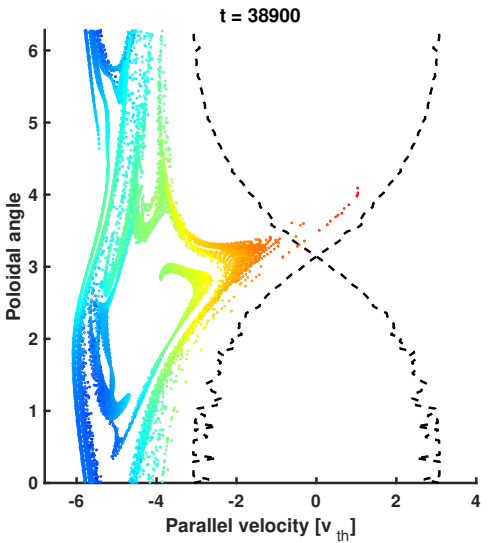

(b)

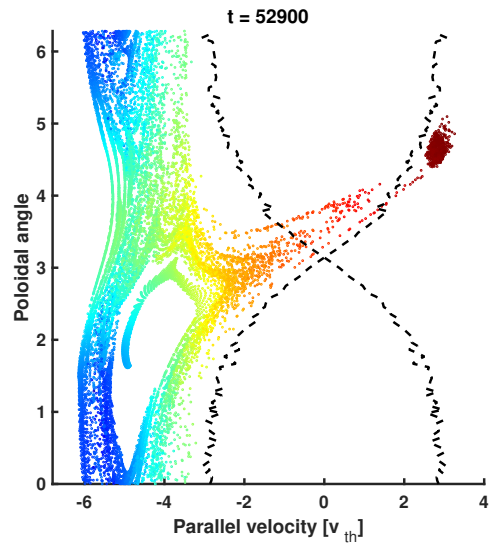

(c)

Figure 11: Position of particles in $\left(v_{\|}, \theta\right)$ space at three different instants: before (left), just after (middle) and well after (right) the nonlinear saturation of the EGAM.

during the whole nonlinear phase, but it is observed to be maximum when the O-point of the EGAM island and the $\mathrm{X}$-point of the trapping-cone are aligned, which occurs at exactly the EGAM frequency, consistently with the time-Fourier transform plotted in the right panel of figure 10. Note that this picture is slightly different from the general understanding à la Chirikov [37]. It is usually believed that chaos originates from the overlap of two contiguous islands, making it possible for a particle to explore regions inside the two islands. This overlap is likely to be produced in the region where the separatrices are closer, i.e. when the two O-points are aligned with each other. Nevertheless, one has to consider that the separatrix of one island is nothing else but the stable and unstable manifolds of its X-point, which merge into one single manifold when the Hamiltonian system is integrable in the sense of Poincaré. When the system is not integrable (which is our case here), the unstable and stable manifolds of the $\mathrm{X}$-point do not merge into one single surface and they start being folded, leading to the homoclinic tangle. Now, if one considers the Xpoint of the contiguous island, the same picture occurs, resulting in the heteroclinic intersection of the stable manifold of one X-point (that of the trapping cone) and the unstable manifold of the other X-point (that of the EGAM) [38-40]. Finally, and only as a graphical illustration of the already analysed interaction EGAMtrapping cone, we give the three dimensional view of the right panel of figure 11, showing that the losses due 
to EGAM are produced by the complexity of a chaotic system in three dimensions.

\section{Conclusions}

In this paper, we have analysed the impact of EGAMs on the transport of particles in a tokamak. This has been done by means of full- $f$ global gyrokinetic simulations using the multi-species version of the Gysela code. A test-particle tracking post-treatment has been developed to integrate the trajectories of passive gyro-centres using the self-consistent electrostatic potential obtained from GYSELA simulations where energetic particles have been introduced to excite EGAMs. We have verified the linear excitation of EGAMs against analytic predictions and shown a good agreement in terms of frequency and growth rate. Also, the position of the different poloidal harmonics in phase space has been verified. We have shown that the flattening of the distribution function in velocity space occurs with the formation of an island in $\left(v_{\|}, \theta\right)$ space, centred around the main EGAM resonant velocity, with additional islands formed in phase space related to different poloidal harmonics $m>1$. We have shown that EGAMs induce particle losses and we have for the first time evidenced that these losses are due to the deformation and interaction between the separatrix of the EGAM island and the X-point of the trapping cone, creating in nonlinear regimes a chaotic channel of transport from $v_{\|}<0$ to $v_{\|}>0$ regions, leading to losses of counter-passing particles at the EGAM frequency. The fact that only counter-passing energetic particles are significantly affected and eventually lost, opens new perspectives for further analysis to optimize the injection of energetic particles in order to minimize the losses.

\section{Acknowledgments}

This work has been carried out thanks to the support of the $A^{*}$ MIDEX project (no. ANR11-IDEX-0001-02) funded by the Investissements d'Avenir French Government program, managed by the French National Research Agency (ANR). This work has also been carried out within the framework of the EUROfusion Consortium and has received funding from the Euratom research and training programme 2014-2018 under grant agreement No 633053. The views and opinions expressed herein do not necessarily reflect those of the European Commission. This work was granted access to the HPC resources of TGCC under the allocation t2016057653 and to the HPC resources of CINES under the allocation A0020507653 made by GENCI
(Grand Equipement National de Calcul Intensif). This work has been done within the framework of the Nonlinear energetic particle dynamics (NLED) European Enabling Research Project (EUROFUSION AWP15-ER-01/ENEA-03) and within the framework of the Verification and development of new algorithms for gyrokinetic codes European Enabling Research Project (EUROFUSION AWP15-ER-01/IPP-01).

\section{Appendix A. Geometry basics: tensor calculus and toroidal geometry}

Note that we give here only a brief introduction to the geometry basics. This is done for the sake of completeness. Nevertheless, the reader can find more material in textbooks such as Ref. [41].

A vector field $\mathbf{A}$ can be expressed by its components in terms of a basis set, which is a linearly independent set of vectors spanning the set of directions. In Cartesian coordinates, the basis set is composed of a set of mutually orthogonal unit and constant vectors so that the vector field $\mathbf{A}$ is decomposed as

$\mathbf{A}=A_{x} \mathbf{e}_{x}+A_{y} \mathbf{e}_{y}+A_{z} \mathbf{e}_{z}$

In a more general way, we can use a basis set built upon a set of three vector fields $\left\{x^{i}\right\}_{i=1,2,3}$ that span the set of directions at each point $(x, y, z)$. The covariant representation of any vector field $\mathbf{A}$ is written as

$\mathbf{A}=A_{i} \nabla x^{i}$

where the covariant component is given by the product

$A_{i}=\mathcal{J}_{x} \frac{\epsilon_{i j k}}{2}\left(\nabla x^{j} \times \nabla x^{k}\right) \cdot \mathbf{A}$

with $\mathcal{J}_{x}=\left[\left(\nabla x^{1} \times \nabla x^{2}\right) \cdot \nabla x^{3}\right]^{-1}$ the Jacobian in space and $\epsilon_{i j k}$ the covariant components of the LeviCivita tensor. The term covariant comes from the transformation properties of the components. To express the vector in a different covariant system $\tilde{x}^{i}$ we use the chain rule for the derivatives as follows

$\mathbf{A}=A_{i} \nabla x^{i}=A_{i} \frac{\partial x^{i}}{\partial \tilde{x}^{j}} \nabla \tilde{x}^{j}$

which gives straightforwardly the covariant components in the basis $\nabla \tilde{x}^{i}$

$\tilde{A}_{j}=A_{i} \frac{\partial x^{i}}{\partial \tilde{x}^{j}}$

Since the new components vary with the basis vectors in the sense that $x^{i}$ is in the numerator, this representations is called covariant. However, the contravariant components vary against the basis vectors in the sense that $x^{i}$ appears in the denominator. The contravariant components are obtained by scalar product

$A^{i}=\mathbf{A} \cdot \nabla x^{i}$ 


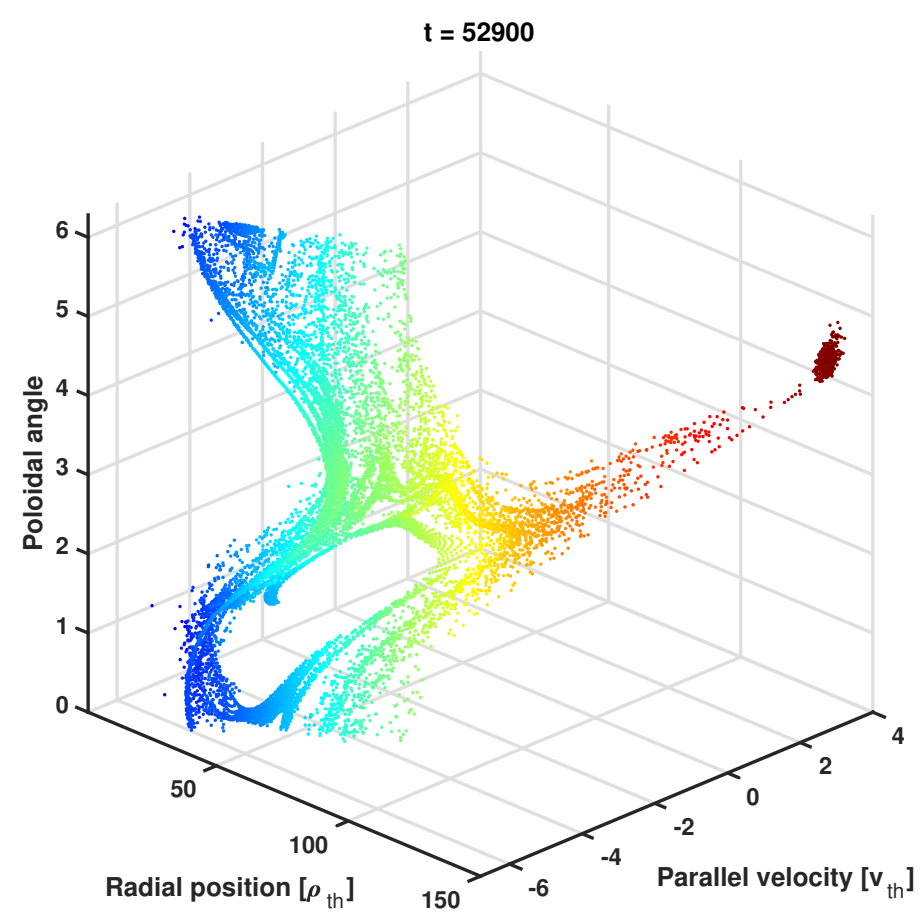

Figure 12: Three dimensional view of the position of test gyro-centres in phase space well after the nonlinear saturation of the EGAM. have

Applying the chain rule for the derivatives, we

$A^{i}=\mathbf{A} \cdot \nabla \tilde{x}^{j} \frac{\partial x^{i}}{\partial \tilde{x}^{j}}$

which gives

$\tilde{A}^{j}=A^{i} \frac{\partial \tilde{x}^{j}}{\partial x^{i}}$

The contravariant vectors can be built using expression A.6, which gives

$\nabla x^{i}=\frac{1}{\mathcal{J}_{x}} \frac{\epsilon_{i j k}}{2} \nabla x^{j} \times \nabla x^{k}$

The relationship between covariant and contravariant components is written

$A^{i}=A_{j} \nabla x^{j} \cdot \nabla x^{i}=g^{i j} A_{j}$

where $g^{i j}$ are the contravariant components of the metric tensor

$g^{i j}=\nabla x^{i} \cdot \nabla x^{j}$

and the covariant tensor is given by the inverse of the contravariant tensor and satisfies

$\operatorname{det}\left(g_{i j}\right)=\mathcal{J}_{x}^{2}$

The gradient of a scalar field $A$ can be written as

$\nabla A=\frac{\partial A}{\partial x^{i}} \nabla x^{i}$ and the contravariant components of the rotational of a vector field $\mathbf{A}$ are expressed as

$(\nabla \times \mathbf{A})^{i}=\frac{1}{\mathcal{J}_{x}} \epsilon^{i j k} \frac{\partial A_{k}}{\partial x^{j}}$

We will use the toroidal coordinate system, i.e. the set of coordinates $\left(x^{1}, x^{2}, x^{3}\right)$ is reduced to the radial position and the poloidal and toroidal angles

$\left(x^{1}, x^{2}, x^{3}\right)=(r, \theta, \varphi)$

where $r$ is the radial position, $\theta$ is the poloidal angle and $\varphi$ is the toroidal angle. The metric tensor is given by its contravariant components as

$g^{i j}=\left(\begin{array}{ccc}|\nabla r|^{2} & \nabla r \cdot \nabla \theta & 0 \\ \nabla \theta \cdot \nabla r & |\nabla \theta|^{2} & 0 \\ 0 & 0 & |\nabla \varphi|^{2}\end{array}\right)$

are then $g^{11} \equiv g^{r r}=1, g^{22} \equiv g^{\theta \theta}=r^{-2}$, $g^{33} \equiv g^{\varphi \varphi}=R^{-2}$ and $g^{i j}=0$ for all $i \neq j$. The major radius of the tokamak is given by

$R(r, \theta)=R_{0}+r \cos \theta$

We use simplified magnetic geometry, with poloidal cross-sections of the magnetic surfaces as circular and concentric. In this case $\nabla r \cdot \nabla \theta=\nabla \theta \cdot \nabla r=$ 0 and the metric tensor is diagonal with $g^{11} \equiv g^{r r}=1$, $g^{22} \equiv g^{\theta \theta}=r^{-2}$ and $g^{33} \equiv g^{\varphi \varphi}=R^{-2}$. Therefore, the 
Jacobian in space is simply $\mathcal{J}_{x}=r R$. The magnetic field is written in the simplified form

$\mathbf{B}=\frac{B_{0} R_{0}}{R}\left(\zeta \mathbf{e}_{\theta}+\mathbf{e}_{\varphi}\right)$

with $B_{0}$ the magnetic field at the magnetic axis, $R_{0}$ the major radius at the magnetic axis, $R$ the major radius at a given position $R=R_{0}+r \cos \theta, \mathbf{e}_{\theta}=r \nabla \theta$, $\mathbf{e}_{\varphi}=R \nabla \varphi$ are the unit vectors in the poloidal and toroidal directions, respectively, and

$\zeta=\frac{r}{q R_{0}}$

where $q$ is the safety factor, which measures the helicity of the magnetic field lines. The covariant and contravariant components of the magnetic field are calculated using expressions A.3 and A.6, respectively, which leads to

$B^{r}=0, B^{\theta}=\frac{B_{0}}{q R}, \quad B^{\varphi}=\frac{B_{0} R_{0}}{R^{2}}$

$B_{r}=0, B_{\theta}=\frac{B_{0} r^{2}}{q R}, B_{\varphi}=B_{0} R_{0}$

\section{Appendix B. Explicit expression of the equations of motion in toroidal coordinates}

In this appendix we will calculate explicitly the expressions of the differential operators in the simplified toroidal geometry of Gysela. First we will give the form of the curvature terms in expressions 10 and 11. We will then derive the equations for the spatial coordinates $(r, \theta$ and $\varphi)$ and for the parallel velocity $v_{\|}$

\section{Appendix B.1. Curvature terms}

We need to calculate $(\nabla \times \mathbf{B}) \cdot \nabla x^{i}$, the operator $(\nabla \times \mathbf{B}) \cdot \nabla$ and $\mathbf{b} \cdot \nabla \times \mathbf{b}$.

The first term is calculated as

$$
(\nabla \times \mathbf{B}) \cdot \nabla x^{i}=\frac{1}{\mathcal{J}_{x}} \epsilon^{l j k} \partial_{j} B_{k} \partial_{l} x^{i}
$$

The radial component $i=r$ can be written as

$(\nabla \times \mathbf{B}) \cdot \nabla r=\frac{1}{\mathcal{J}_{x}} \epsilon^{r \theta \varphi}\left(\partial_{\theta} B_{\varphi}-\partial_{\varphi} B_{\theta}\right)$

Taking into account that the equilibrium magnetic field is axisymmetric and that $\partial_{\theta} B_{\varphi}=0$ we have

$(\nabla \times \mathbf{B}) \cdot \nabla r=0$

The poloidal component $i=\theta$ can be written as

$(\nabla \times \mathbf{B}) \cdot \nabla \theta=\frac{1}{\mathcal{J}_{x}} \epsilon^{\theta r \varphi}\left(\partial_{r} B_{\varphi}-\partial_{\varphi} B_{r}\right)$

Since $B_{\varphi}=$ cst and the magnetic field does not have any radial component we have

$(\nabla \times \mathbf{B}) \cdot \nabla \theta=0$
The toroidal component $i=\varphi$ can be written as

$(\nabla \times \mathbf{B}) \cdot \nabla \varphi=\frac{1}{\mathcal{J}_{x}} \epsilon^{\varphi r \theta}\left(\partial_{r} B_{\theta}-\partial_{\theta} B_{r}\right)$

Since the magnetic field does not have any radial component, we have

$(\nabla \times \mathbf{B}) \cdot \nabla \varphi=\frac{1}{r R} \frac{\partial B_{\theta}}{\partial r}$

Following the previous calculation the second term is calculated using only the toroidal component $i=\varphi$. However, since all the modes we are considering are axisymmetric, this term can be straightfowardly not considered when integrating the equations of motion.

The third term is calculated as

$\mathbf{b} \cdot \nabla \times \mathbf{b}=\frac{1}{\mathcal{J}_{x}} \epsilon^{i j k} b_{i} \partial_{j} b_{k}$

Taking into account that the magnetic field does not have any radial component, we have

$\mathbf{b} \cdot \nabla \times \mathbf{b}=\frac{1}{\mathcal{J}_{x}}\left(b_{\varphi} \partial_{r} b_{\theta}-b_{\theta} \partial_{r} b_{\varphi}\right)$

Using the fact that $b_{\varphi}=B^{-1} B_{\varphi}$ and $b_{\theta}=B^{-1} B_{\theta}$ and taking into account that $B_{\varphi}=$ cst we finally have

$\mathbf{b} \cdot \nabla \times \mathbf{b}=\frac{1}{r R} \frac{B_{\varphi}}{B^{2}} \frac{\partial B_{\theta}}{\partial r}$

This expression will be used to calculate $B_{\|, s}^{*}$.

Appendix B.2. Contravariant components of the drifts

The contravariant components of the drifts in toroidal coordinates are calculated as follows. The radial contravariant component of the $\mathbf{E} \times \mathbf{B}$ drift is calculated using expressions $7 a$ and 8

$\mathbf{v}_{E} \cdot \nabla r=\frac{1}{B_{\|, s}^{*}} \frac{1}{r R} \epsilon^{i r k} b_{k} \partial_{i} \bar{\phi}$

where $\bar{\phi} \equiv J_{0} \phi$. Taking into account that we consider only axi-symmetric modes, then $i \equiv \theta$ and therefore $k \equiv \varphi$. The covariant component of the unit vector along the magnetic field lines can be written in terms of the covariant component of the magnetic field as $b_{\varphi}=B_{\varphi} / B$. Using expression A.20 we can write

$b_{\varphi}=R_{0} / B$

The contravariant component of the $\mathbf{E} \times \mathbf{B}$ drift can be finally written as

$\mathbf{v}_{E} \cdot \nabla r=-\frac{1}{B_{\|, s}^{*} \sqrt{1+\zeta^{2}}} \frac{1}{r} \frac{\partial \bar{\phi}}{\partial \theta}$

Using again expressions $7 a$ and 8 and performing similar calculations one can find the poloidal contravariant component

$\mathbf{v}_{E} \cdot \nabla \theta=\frac{1}{B_{\|, s}^{*} \sqrt{1+\zeta^{2}}} \frac{1}{r} \frac{\partial \bar{\phi}}{\partial r}$ 
The radial contravariant component of the magnetic drift is calculated using expressions $7 a$ and 8

$\mathbf{v}_{D} \cdot \nabla r=\frac{v_{\|}^{2}+\mu B}{Z_{s} B_{\|, s}^{*} B} \frac{1}{r R} \epsilon^{i r k} b_{k} \partial_{i} B$

Since the magnetic field has only poloidal and toroidal components and does not depend on $\varphi$, the only term in the sum is $b_{\varphi} \partial_{\theta} B$. Therefore, we can write

$\mathbf{v}_{D} \cdot \nabla r=\frac{v_{\|}^{2}+\mu B}{Z_{s} B_{\|, s}^{*} B} \frac{1}{r R} \epsilon^{\theta r \varphi} b_{\varphi} \partial_{\theta} B$

The covariant component $b_{\varphi}$ has been calculated before. We can therefore write the radial contravariant component of the magnetic drift as

$\mathbf{v}_{D} \cdot \nabla r=-\frac{v_{\|}^{2}+\mu B}{Z_{s} B_{\|, s}^{*} B} \frac{1}{\sqrt{1+\zeta^{2}}} \frac{1}{r} \frac{\partial B}{\partial \theta}$

The poloidal contravariant component of the magnetic field is calculated in the same way and reads

$\mathbf{v}_{D} \cdot \nabla \theta=\frac{v_{\|}^{2}+\mu B}{Z_{s} B_{\|, s}^{*} B} \frac{1}{\sqrt{1+\zeta^{2}}} \frac{1}{r} \frac{\partial B}{\partial r}$

Note that the contravariant toroidal components of the drifts vanish for axisymmetric modes.

\section{Appendix B.3. Parallel gradients}

Based on the previous results of the calculation of $\nabla \times$ $\mathbf{B}$, the parallel gradient of $r$ does not contribute to the evolution of $r$. For the evolution of the poloidal angle, only the projection of the gradient on $\mathbf{b}$ contributes

$\frac{1}{\sqrt{A_{s}}} v_{\|} \mathbf{b}_{s}^{*} \cdot \nabla \theta=\frac{1}{\sqrt{A_{s}}} \frac{1}{B_{\|, s}^{*} / B \sqrt{1+\zeta^{2}}} \frac{1}{q R_{0}} v_{\|}$

where $B_{\|, s}^{*} / B=\left(1+\sqrt{A_{s}} / Z_{s} v_{\|} / B \mathbf{b} \cdot \nabla \times \mathbf{b}\right)$.

As for the toroidal angle, we have

$$
\begin{array}{r}
\frac{1}{\sqrt{A_{s}}} v_{\|} \mathbf{b}_{s}^{*} \cdot \nabla \varphi=\frac{1}{\sqrt{A_{s}}} v_{\|}\left(\frac{1}{B_{\|, s}^{*} / B \sqrt{1+\zeta^{2}}} \frac{1}{R_{0}}\right. \\
\left.+\frac{m_{s} v_{\|}}{e Z_{s} B_{\|, s}^{*} B} \frac{1}{r R} \frac{\partial B_{\theta}}{\partial r}\right)
\end{array}
$$

The parallel gradients of any axisymmetric field $A$, where $A$ can be either the magnetic field or the electrostatic potential, can be calculated as follows

$\mathbf{b}_{s}^{*} \cdot \nabla A=\mathbf{b}_{s}^{*} \cdot \nabla x^{i} \partial_{i} A=\mathbf{b}_{s}^{*} \cdot \nabla r \partial_{r} A+\mathbf{b}_{s}^{*} \cdot \nabla \theta \partial_{\theta} A(\mathrm{~B} .20)$

where the contravariant components of $\mathbf{b}_{s}^{*}$ were already calculated.

$\mathbf{b}_{s}^{*} \cdot \nabla A=\frac{1}{B_{\|, s}^{*} / B \sqrt{1+\zeta^{2}}} \frac{1}{q R_{0}} \partial_{\theta} A$
Therefore, the two first terms in the right hand side of equation $9 b$ can be written as

$$
\begin{aligned}
-\frac{\mu}{\sqrt{A_{s}}} \mathbf{b}_{s}^{*} \cdot \nabla B & = \\
& -\frac{\mu}{\sqrt{A_{s}}} \frac{1}{B_{\|, s}^{*} / B \sqrt{1+\zeta^{2}}} \frac{1}{q R_{0}} \frac{\partial B}{\partial \theta} \\
-\frac{Z_{s}}{\sqrt{A_{s}}} \mathbf{b}_{s}^{*} \cdot \nabla \bar{\phi} & = \\
& -\frac{Z_{s}}{\sqrt{A_{s}}} \frac{1}{B_{\|, s}^{*} / B \sqrt{1+\zeta^{2}}} \frac{1}{q R_{0}} \frac{\partial \bar{\phi}}{\partial \theta}
\end{aligned}
$$

The third term of the right hand side of equation $9 b, \mathbf{E} \times \mathbf{B}$, due to the curvature is calculated using a generalized expression of $7 a$

$\mathbf{v}_{E} \cdot \nabla B=\frac{1}{B_{\|, s}^{*}} \mathbf{b} \cdot(\nabla \bar{\phi} \times \nabla B)$

which gives the expression for the third term as follows

$\frac{v_{\|}}{B} \mathbf{v}_{E} \cdot \nabla B=\frac{v_{\|}}{B B_{\|, s}^{*}} \frac{1}{\sqrt{1+\zeta^{2}}} \frac{1}{r}\left(\frac{\partial \bar{\phi}}{\partial r} \frac{\partial B}{\partial \theta}-\frac{\partial \bar{\phi}}{\partial \theta} \frac{\partial B}{\partial r}\right)$

\section{Appendix B.4. Final set of equations of motion}

The time evolution of the coordinates $\left(r, \theta, \varphi, v_{\|}\right)$is thus written as follows

$$
\begin{aligned}
\alpha \frac{\mathrm{d} r}{\mathrm{~d} t}= & -\frac{1}{B} \frac{1}{r} \frac{\partial \bar{\phi}}{\partial \theta}-\frac{v_{\|}^{2}+\mu B}{Z_{s} B^{2}} \frac{1}{r} \frac{\partial B}{\partial \theta} \\
\alpha \frac{\mathrm{d} \theta}{\mathrm{d} t}= & \frac{1}{\sqrt{A_{s}}} \frac{1}{q R_{0}} v_{\|}+\frac{1}{B} \frac{1}{r} \frac{\partial \bar{\phi}}{\partial r}+\frac{v_{\|}^{2}+\mu B}{Z_{s} B^{2}} \frac{1}{r} \frac{\partial B}{\partial r} \\
\alpha \frac{\mathrm{d} \varphi}{\mathrm{d} t}= & \frac{1}{\sqrt{A_{s}}} \frac{1}{R_{0}} v_{\|}+\alpha \frac{v_{\|}^{2}}{Z_{s} B_{\|, s}^{*} B} \frac{1}{r R} \frac{\partial}{\partial r}\left(\frac{r^{2}}{q R}\right) \\
\alpha \frac{\mathrm{d} v_{\|}}{\mathrm{d} t}= & -\frac{\mu}{\sqrt{A_{s}}} \frac{1}{q R_{0}} \frac{\partial B}{\partial \theta}-\frac{Z_{s}}{\sqrt{A_{s}}} \frac{1}{q R_{0}} \frac{\partial \bar{\phi}}{\partial \theta} \\
& +\frac{v_{\|}}{B^{2}} \frac{1}{r}\left(\frac{\partial \bar{\phi}}{\partial r} \frac{\partial B}{\partial \theta}-\frac{\partial \bar{\phi}}{\partial \theta} \frac{\partial B}{\partial r}\right)
\end{aligned}
$$

where $\alpha=B_{\|, s}^{*} / B \sqrt{1+\zeta^{2}}$

\section{Appendix C. Conservation properties}

The set of equations derived in the previous section conserves energy (for the unperturbed trajectories, i.e. for $\phi=0$ ) and toroidal canonical momentum (even if $\phi \neq 0$ as long as the modes are axisymmetric). The toroidal canonical momentum is defined, using normalized quantities, as

$P_{\varphi}=-Z_{s} \psi+\sqrt{A_{s}} \frac{B_{\varphi}}{B} v_{\|}$

where $\psi$ is the poloidal flux defined as

$\psi(r)=\int_{0}^{r} \frac{r^{\prime}}{q\left(r^{\prime}\right)} \mathrm{d} r^{\prime}$ 
where $r$ and $r^{\prime}$ are normalized distances. Note that with this definition $\mathrm{d} \psi / \mathrm{d} r>0$. The total time derivative reads

$\frac{\mathrm{d} P_{\varphi}}{\mathrm{d} t}=-Z_{s} \frac{\mathrm{d} \psi}{\mathrm{d} t}+\sqrt{A_{s}} \frac{\mathrm{d} b_{\varphi}}{\mathrm{d} t} v_{\|}+\sqrt{A_{s}} b_{\varphi} \frac{\mathrm{d} v_{\|}}{\mathrm{d} t}$

The time derivative of the poloidal flux is written, up to a multiplying factor introducing $B_{\|, s}^{*}$ effects, as

$\frac{\mathrm{d} \psi}{\mathrm{d} t}=\frac{r}{q}\left(-\frac{1}{B} \frac{1}{r} \frac{\partial \bar{\phi}}{\partial \theta}+\frac{v_{\|}^{2}+\mu B}{Z_{s} B^{2}} \frac{1}{r} \frac{R_{0}}{R^{2}} \sqrt{1+\zeta^{2}} \frac{\partial R}{\partial \theta}\right)$

where the partial derivative of $B$ with respect to $\theta$ has been expressed in terms of the partial derivative of $R$ with respect to $\theta$. The time derivative of $B_{\varphi} / B$ is $\mathrm{d}_{t} b_{\varphi}=\mathrm{d}_{t}\left(R / \sqrt{1+\zeta^{2}}\right)$. The time derivative of the major radius is written as $\mathrm{d}_{t} R=\partial_{r} R \mathrm{~d}_{t} r+\partial_{\theta} R \mathrm{~d}_{t} \theta$ Expressing the total time derivatives of $r$ and $\theta$ and the partial derivatives of $B$ as a function of the partial derivatives of $R$ we can write, up to a multiplying factor introducing the $B_{\|, s}^{*}$ effects, $\mathrm{d}_{t} b_{\varphi}=\left(\mathrm{d}_{t} b_{\varphi}\right)_{1}+\left(\mathrm{d}_{t} b_{\varphi}\right)_{2}+$ $\left(\mathrm{d}_{t} b_{\varphi}\right)_{3}$, where

$$
\begin{aligned}
\alpha_{1}\left(\frac{\mathrm{d} b_{\varphi}}{\mathrm{d} t}\right)_{1}= & -\frac{1}{B} \frac{1}{r} \frac{\partial \bar{\phi}}{\partial \theta} \\
& +\frac{v_{\|}^{2}+\mu B}{Z_{s} B^{2}} \frac{1}{r} \frac{R_{0}}{R^{2}} \sqrt{1+\zeta^{2}} \frac{\partial R}{\partial \theta} \\
\alpha_{2}\left(\frac{\mathrm{d} b_{\varphi}}{\mathrm{d} t}\right)_{2}= & \frac{1}{\sqrt{A_{s}}} \frac{1}{q R_{0}} v_{\|}+\frac{1}{B} \frac{1}{r} \frac{\partial \bar{\phi}}{\partial r} \\
& -\frac{v_{\|}^{2}+\mu B}{Z_{s} B^{2}} \frac{1}{r} \frac{R_{0}}{R^{2}} \sqrt{1+\zeta^{2}} \frac{\partial R}{\partial r} \\
& +\frac{v_{\|}^{2}+\mu B}{Z_{s} B^{2}} \frac{1}{r} \frac{1}{R} \frac{\zeta}{\sqrt{1+\zeta^{2}}} \frac{\mathrm{d} r / q}{\mathrm{~d} r} \\
\alpha_{3}\left(\frac{\mathrm{d} b_{\varphi}}{\mathrm{d} t}\right)_{3}= & -\frac{1}{B} \frac{1}{r} \frac{\partial \bar{\phi}}{\partial \theta} \\
& +\frac{v_{\|}^{2}+\mu B}{Z_{s} B^{2}} \frac{1}{r} \frac{R_{0}}{R^{2}} \sqrt{1+\zeta^{2}} \frac{\partial R}{\partial \theta}
\end{aligned}
$$

with

$\alpha_{1}^{-1}=\frac{1}{\sqrt{1+\zeta^{2}}} \frac{\partial R}{\partial r}$

$\alpha_{2}^{-1}=\frac{1}{\sqrt{1+\zeta^{2}}} \frac{\partial R}{\partial \theta}$

$\alpha_{3}^{-1}=-\frac{1}{\sqrt{1+\zeta^{2}}} \frac{R}{R_{0}} \frac{\zeta}{1+\zeta^{2}} \frac{\mathrm{d} r / q}{\mathrm{~d} r}$

Finally, the last term of equation C.3 is written, up to a multiplying factor introducing the $B_{\|, s}^{*}$ effects, as $b_{\varphi} \mathrm{d}_{t} v_{\|}=\left(b_{\varphi} \mathrm{d}_{t} v_{\|}\right)_{1}+\left(b_{\varphi} \mathrm{d}_{t} v_{\|}\right)_{2}+\left(b_{\varphi} \mathrm{d}_{t} v_{\|}\right)_{3}$, where the first term includes the radial derivative of $B$, the second term includes the poloidal derivative of $B$ and the thirm term does not include any derivative of $B$, i.e.

$$
\begin{aligned}
\left(b_{\varphi} \mathrm{d}_{t} v_{\|}\right)_{1}= & \frac{1}{\sqrt{1+\zeta^{2}}} R\left(\frac{\mu}{\sqrt{A_{s}}} \frac{1}{q R_{0}}\right. \\
& \left.-\frac{v_{\|}}{B^{2}} \frac{1}{r} \frac{\partial \bar{\phi}}{\partial r}\right) \frac{R_{0}}{R^{2}} \sqrt{1+\zeta^{2}} \frac{\partial R}{\partial \theta} \\
\left(b_{\varphi} \mathrm{d}_{t} v_{\|}\right)_{2}= & \frac{1}{\sqrt{1+\zeta^{2}}} R \frac{v_{\|}}{B^{2}} \frac{1}{r} \frac{\partial \bar{\phi}}{\partial \theta}\left(\frac{R_{0}}{R^{2}} \sqrt{1+\zeta^{2}} \frac{\partial R}{\partial r}\right. \\
& \left.-\frac{1}{R} \frac{\zeta}{\sqrt{1+\zeta^{2}}} \frac{\mathrm{d} r / q}{\mathrm{~d} r}\right) \\
\left(b_{\varphi} \mathrm{d}_{t} v_{\|}\right)_{3}= & -\frac{1}{\sqrt{1+\zeta^{2}}} R \frac{Z_{s}}{\sqrt{A_{s}}} \frac{1}{q R_{0}} \frac{\partial \bar{\phi}}{\partial \theta}
\end{aligned}
$$

Introducing equations C.4 to C.13 in equation C.3, using the expression of $B=R_{0} \sqrt{1+\zeta^{2}} / R$ and rearranging the terms, we can prove that the toroidal canonical momentum is an exact invariant for axisymmetric modes, i.e.

$\frac{\mathrm{d} P_{\varphi}}{\mathrm{d} t}=0$

The kinetic energy (total energy in the absence of electrostatic potential) reads $E=v_{\|}^{2} / 2+\mu B$. Therefore, under the hypothesis of adiabatic invariance of $\mu$, the time derivative is written as $\mathrm{d}_{t} E=v_{\|} \mathrm{d}_{t} v_{\|}+$ $\mu\left(\partial_{r} B \mathrm{~d}_{t} r+\partial_{\theta} B \mathrm{~d}_{t} \theta\right)$. Using the equations of motion with $\bar{\phi}=0$ we can prove the conservation of energy

$$
\begin{aligned}
\frac{\mathrm{d} E}{\mathrm{~d} t}= & v_{\|}\left(-\frac{\mu}{\sqrt{A_{s}}} \frac{1}{q R_{0}} \frac{\partial B}{\partial \theta}\right) \\
& +\mu\left(-\frac{v_{\|}^{2}+\mu B}{Z_{s} B^{2}} \frac{1}{r} \frac{\partial B}{\partial \theta}\right) \frac{\partial B}{\partial r} \\
& +\mu\left(\frac{1}{\sqrt{A_{s}}} \frac{1}{q R_{0}} v_{\|}+\frac{v_{\|}^{2}+\mu B}{Z_{s} B^{2}} \frac{1}{r} \frac{\partial B}{\partial r}\right) \frac{\partial B}{\partial \theta} \\
& =0
\end{aligned}
$$

[1] D Zarzoso, A Biancalani, A Bottino, Ph Lauber, E Poli, JB Girardo, X Garbet, and RJ Dumont. Nuclear Fusion, 54(10):103006, 2014.

[2] Jean-Baptiste Girardo, David Zarzoso, Rémi Dumont, Xavier Garbet, Yanick Sarazin, and Sergei Sharapov. Physics of Plasmas, 21(9):092507, 2014.

[3] N. Winsor, J.L. Johnson, and J.M. Dawson. Geodesic acoustic waves in hydromagnetic systems. Physics of Fluids, 11:2448, 1968.

[4] GY Fu. Energetic-particle-induced geodesic acoustic mode. Physical review letters, 101(18):185002, 2008.

[5] CJ Boswell, HL Berk, DN Borba, T. Johnson, SD Pinches, and SE Sharapov. Physics Letters A, 358(2):154-158, 2006.

[6] HL Berk, CJ Boswell, D Borba, ACA Figueiredo, T Johnson, MFF Nave, SD Pinches, SE Sharapov, et al. Nuclear fusion, 46(10):S888, 2006. 
[7] R. Nazikian, GY Fu, ME Austin, HL Berk, RV Budny, NN Gorelenkov, WW Heidbrink, CT Holcomb, GJ Kramer, GR McKee, et al. Intense geodesic acousticlike modes driven by suprathermal ions in a tokamak plasma. Physical review letters, 101(18):185001, 2008.

[8] RK Fisher, DC Pace, GJ Kramer, MA Van Zeeland, R Nazikian, WW Heidbrink, and M García-Muñoz. Nuclear Fusion, 52(12):123015, 2012.

[9] D. Zarzoso, X. Garbet, Y. Sarazin, R. Dumont, and V. Grandgirard. Physics of Plasmas, 19(2):022102022102, 2012.

[10] D Zarzoso, Y Sarazin, X Garbet, R Dumont, A Strugarek, J Abiteboul, $\mathrm{T}$ Cartier-Michaud, G Dif-Pradalier, $\mathrm{Ph}$ Ghendrih, V Grandgirard, et al. Physical Review Letters, 110(12):125002, 2013.

[11] Alessandro Biancalani, Alberto Bottino, Ph Lauber, and David Zarzoso. Nuclear Fusion, 54(10):104004, 2014.

[12] Hao Wang, Yasushi Todo, Charlson C Kim, et al. Physical review letters, 110(15):155006, 2013.

[13] Hao Wang, , Yasushi Todo, , Takeshi Ido, , Masaki Osakabe, and . Physics of Plasmas, 22(9):092507, 2015.

[14] D Zarzoso, P Migliano, V Grandgirard, G Latu, and C Passeron. Nucl. Fusion, 57(072011):072011, 2017.

[15] HL Berk and T. Zhou. Nuclear Fusion, 50:035007, 2010.

[16] Z. Qiu, F. Zonca, and L. Chen. Plasma Physics and Controlled Fusion, 52:095003, 2010.

[17] Makoto Sasaki, Kimitaka Itoh, and SI Itoh. Plasma Physics and Controlled Fusion, 53(8):085017, 2011.

[18] Haijun Ren and Chao Dong. Physics of Plasmas, 21(10):102506, 2014.

[19] ZS Qu, MJ Hole, and M Fitzgerald. Physical review letters, 116(9):095004, 2016.

[20] M Sasaki, N Kasuya, K Itoh, K Hallatschek, M Lesur, Y Kosuga, and S-I Itoh. Physics of Plasmas, 23(10):102501, 2016.

[21] Haijun Ren. Nuclear Fusion, 57(1):016023, 2016.

[22] ZS Qu, MJ Hole, and M Fitzgerald. Plasma Physics and Controlled Fusion, 59(5):055018, 2017.

[23] M Garcia-Munoz, H-U Fahrbach, H Zohm, and ASDEX Upgrade Team. Review of Scientific Instruments, 80(5):053503, 2009.

[24] GJ Kramer, RV Budny, A Bortolon, ED Fredrickson, GY Fu, WW Heidbrink, R Nazikian, E Valeo, and MA Van Zeeland. Plasma Physics and Controlled Fusion, 55(2):025013, 2013.

[25] Ya I Kolesnichenko, VV Lutsenko, Yu V Yakovenko, BS Lepiavko, B Grierson, WW Heidbrink, and R Nazikian. Plasma Physics and Controlled Fusion, 58(4):045024, 2016.

[26] Virginie Grandgirard, Jérémie Abiteboul, Julien Bigot, Thomas Cartier-Michaud, Nicolas Crouseilles, Guilhem Dif-Pradalier, Ch Ehrlacher, Damien Esteve, Xavier Garbet, Ph Ghendrih, et al. Computer Physics Communications, 207:35-68, 2016.

[27] RJ Dumont, D Zarzoso, Y Sarazin, X Garbet, A Strugarek, J Abiteboul, T Cartier-Michaud, G Dif-Pradalier, $\mathrm{Ph}$ Ghendrih, JB Girardo, et al. Plasma Physics and Controlled Fusion, 55(12):124012, 2013.

[28] S Jolliet, Alberto Bottino, P Angelino, Roman Hatzky, Trach-Minh Tran, BF McMillan, O Sauter, K Appert, Yasuhiro Idomura, and Laurent Villard. A global collisionless pic code in magnetic coordinates. Computer Physics Communications, 177(5):409-425, 2007.

[29] A Biancalani, I Chavdarovski, Z Qiu, A Bottino, D Del Sarto, A Ghizzo, Ö Gürcan, P Morel, and I Novikau. Saturation of energetic-particledriven geodesic acoustic modes due to wave-particle nonlinearity. Journal of Plasma Physics, 83(6), 2017.

[30] A Biancalani, I Chavdarovski, Z Qiu, A Bottino, D Del Sarto, A Ghizzo, O Gurcan, P Morel, and
I Novikau. Nonlinear gyrokinetic investigation of energetic-particle-driven geodesic acoustic modes. 17th European Fusion Theory Conference, Athens, Greece.

[31] RGL Vann, RO Dendy, G Rowlands, TD Arber, and $\mathrm{N}$ dAmbrumenil. Fully nonlinear phenomenology of the berk-breizman augmentation of the vlasov-maxwell system. Physics of Plasmas, 10(3):623-630, 2003.

[32] M Lesur and Y Idomura. Nonlinear categorization of the energetic-beam-driven instability with drag and diffusion. Nuclear Fusion, 52(9):094004, 2012.

[33] A Biancalani, N Carlevaro, A Bottino, G Montani, and Z Qiu. Nonlinear velocity redistribution caused by energetic-particle-driven geodesic acoustic modes, mapped with the beam-plasma system. in preparation, 2018.

[34] Donald Shepard. In Proceedings of the 1968 23rd ACM national conference, pages 517-524. ACM, 1968.

[35] Xi Chen, ME Austin, RK Fisher, WW Heidbrink, GJ Kramer, R Nazikian, DC Pace, CC Petty, and MA Van Zeeland. Enhanced localized energetic-ion losses resulting from single-pass interactions with alfvén eigenmodes. Physical review letters, 110(6):065004, 2013.

[36] RB Zhang, GY Fu, RB White, and XG Wang. Local wave particle resonant interaction causing energetic particle prompt loss in diii-d plasmas. Nuclear Fusion, 55(12):122002, 2015

[37] Boris V Chirikov. Physics reports, 52(5):263-379, 1979.

[38] Yves Elskens and D.F Escande. Physica D: Nonlinear Phenomena, 62(1):66 - 74, 1993.

[39] Allan J Lichtenberg and Michael A Lieberman. Regular and stochastic motion, volume 38. Springer Science \& Business Media, 2013.

[40] Yves Elskens and DF Escande. Microscopic dynamics of plasmas and chaos. CRC Press, 2002.

[41] Richard D Hazeltine and James D Meiss. Plasma confinement. Courier Corporation, 2003. 